\title{
Unstable CMC spheres and outlying CMC spheres in AF 3-manifolds
}

\author{
Shiguang $\mathrm{Ma}^{*}$
}

April 29, 2018

\begin{abstract}
In this paper, we introduce a non-linear ODE method to construct CMC surfaces in Riemannian manifolds with symmetry. As an application we construct unstable CMC spheres and outlying CMC spheres in asymptotically Schwarzschild manifolds with metrics like $g_{i j}=\left(1+\frac{1}{l}\right)^{2} \delta_{i j}+O\left(l^{-2}\right)$. The existence of unstable CMC spheres tells us that the stability condition in Qing-Tian's work 12 can not be removed generally.
\end{abstract}

\section{Introduction}

Constant mean curvature (CMC) surfaces are a kind of important submanifolds. In this paper we mainly focus on the CMC spheres in asymptotically flat (AF) 3-manifolds. First let's state some background works in this area.

In 1996, G. Huisken and S.T. Yau proved the existence of a foliation of stable CMC spheres in asymptotically Schwarzschid manifolds in [5]. They also proved, under certain radius condition, the uniqueness of the stable CMC spheres. In [12, J. Qing and G. Tian removed this radius condition, i.e. they proved that, outside certain compact subset, any stable CMC sphere which separates the compact part from infinity belongs to those constructed in [5. There are many sequential works. Lan-hsuan Huang did similar work as Huisken and Yau in general AF manifolds with RT conditions (those are a series of asymptotically "odd" or "even" conditions). See 4]. In 9, Nerz considered the existence of CMC foliation in AF manifolds without RT conditions. The uniqueness results of Huang and Nerz also need radius conditions. In [6, I proved a uniqueness result under mild radius condition, which improved Huang's uniqueness result in [4] in some special case. In 17, I removed the radius condition in proving the uniqueness in AF manifolds with decay rate of the metic to be -1 . Gang Tian and Andre Neves have also

*School of Mathematical Sciences and LPMC, Nankai University, Tianjin 300071, People's Republic of China, msgdyx8741@nankai.edu.cn. The author was supported by NSFC grant No.11301284, NSFG grant No. 11571185 and "Specialized Research Fund for the Doctoral Program of Higher Education", No.20120031120028. 
considered similar existence and uniqueness problems in asymptotically hyperbolic manifolds in 10 and [11.

However, in all the above works, one has to assume stability of the CMC surfaces to prove the uniqueness ( In Nerz's work he used an integral estimate on the mean curvature instead ). It was asked by Professor Gang Tian that whether one can remove the stability condition in proving the uniqueness and if the answer is no generally, when we can do this. In [1 Simon Brendle prove that in a class of warped product metrics the only CMC spheres are umbilic. In particular, in Schwarzschild manifolds with positive mass, the only embedded CMC surfaces are spheres of symmetry. This theorem requires assumptions on neither the topology nor the stability of the surfaces. Then it is natural to consider asymptotically Schwarzschild manifolds with positive mass. In this paper without loss of generality we consider asymptotically Schwarzschild manifolds with only one asymptotically flat end. Suppose $\left(M, d s^{2}\right)$ is a complete Riemannian manifold. For a compact subset $K \subset M$, we assume $M \backslash K$ is diffeomorphic to $\mathbb{R}^{3} \backslash \bar{B}_{1}(0)$ and we assume $\left\{x_{i}\right\}_{i=1}^{3}$ are the standard coordinates of $\mathbb{R}^{3}$. Denote

$$
l=\sqrt{x_{1}^{2}+x_{2}^{2}+x_{3}^{3}} .
$$

Note that the Schwarzschild metric with mass 1 takes the form

$$
g_{i j}^{S}=\left(1+\frac{1}{2 l}\right)^{4} \delta_{i j}
$$

with $\delta_{i j}$ to be the standard Euclidean metric.

We use the cylindrical coordinates $(x, r, \theta)$ to calculate, namely,

$$
\left\{\begin{array}{l}
x_{1}=x, \\
x_{2}=r \cos \theta, \\
x_{3}=r \sin \theta,
\end{array}\right.
$$

where $r=\sqrt{x_{2}^{2}+x_{3}^{2}}$.

For $\lambda>0$ and $p \in \mathbb{R}$, let $\phi(s)=\phi_{\lambda, p}(s), s \in[0,+\infty]$ be a smooth monotonic function such that

$$
\phi(s)= \begin{cases}1, & s \in[0, \lambda], \\ p, & s \in[2 \lambda,+\infty],\end{cases}
$$

and

$$
\lambda\left|\phi^{\prime}\right|+\lambda^{2}\left|\phi^{\prime \prime}\right| \leq C|p-1| .
$$

We consider asymptotically Schwarzschild metrics which take the following form on $\mathbb{R}^{3} \backslash \bar{B}_{1}(0)$

$$
d s^{2}\left(\partial_{i}, \partial_{j}\right)=\delta_{i j}+T_{i j}
$$

where

$$
\left\{\begin{array}{l}
T_{r r}=g_{1}(r, x)=\frac{2}{l}+\frac{1}{l^{2}}, \\
T_{\theta \theta}=g_{2}(r, x)=r^{2}\left(\frac{2}{l}+\frac{1}{l^{2}} \phi_{\lambda, p}\left(\left|\frac{r}{x}\right|\right)\right), \\
T_{x x}=g_{3}(r, x)=\frac{2}{l}+\frac{1}{l^{2}} \\
T_{r x}=T_{r \theta}=T_{x \theta}=0 .
\end{array}\right.
$$


Note that when $\left|\frac{r}{x}\right|=+\infty$, it should be understood as that $x=0$.

Let

$$
\chi(r, x)=\frac{1+\frac{2}{l}+\frac{1}{l^{2}} \phi_{\lambda, p}\left(\left|\frac{r}{x}\right|\right)}{1+\frac{2}{l}+\frac{1}{l^{2}}} .
$$

So we have $r^{2}+T_{\theta \theta}=r^{2} \chi(r, x)\left(1+g_{1}(r, x)\right)$.

It is obvious that for fixed $\lambda, p, d s^{2}$ is a smooth metric and

$$
d s^{2}=g^{S}+O\left(l^{-2}\right) .
$$

Remark. Note that

$$
\left\{\begin{array}{l}
x_{2}=r \cos \theta \\
x_{3}=r \sin \theta
\end{array}\right.
$$

Assume $x=x_{1}>0$. Let $d s^{2}-\left(1+\frac{1}{l}\right)^{2} \delta_{i j}=S_{i j}$. Then by direct calculation we have

$$
S_{i j, i j}-S_{i i, j j}=-\frac{\frac{2 x \phi^{\prime}\left(\frac{r}{x}\right)}{r}+\phi^{\prime \prime}\left(\frac{r}{x}\right)}{x^{4}} .
$$

For any $0<\alpha<\beta$.

$$
\begin{aligned}
& \int_{\alpha \leq x_{1} \leq \beta}\left(S_{i j, i j}-S_{i i, j j}\right) d x_{1} d x_{2} d x_{3} \\
= & -2 \pi \int_{\alpha}^{\beta} \frac{1}{x^{2}} d x \int_{\lambda}^{2 \lambda}\left(2 \phi^{\prime}(s)+s \phi^{\prime \prime}(s)\right) d s \\
= & 2 \pi\left(\frac{1}{\beta}-\frac{1}{\alpha}\right)(p-1) .
\end{aligned}
$$

So when $p>1, S_{i j, i j}-S_{i i, j j}$ makes a negative contribution to the scalar curvature. Whether the scalar curvature of an asymptotically Schwarzschild manifold is positive is a very delicate thing. When the scalar curvature is negative, we have more flexibility while when the scalar curvature is positive, we have more rigidity. This can also be seen in 2 and 3 .

Now we can state the main theorems of this paper.

Theorem 1.1. Suppose $\left(M \backslash K, d s^{2}\right)$ is an asymptotically flat end with $d s^{2}\left(\partial_{i}, \partial_{j}\right)=$ $\delta_{i j}+T_{i j}$ with $T_{i j}$ given by (3). Then we can fix $\lambda>0$ small and $p>0$ large such that there is $\delta(\lambda, p)>0$ such that for $0<H<\delta(\lambda, p)$, there is an embedded $C M C$ sphere $\Sigma$ which is unstable, has mean curvature $H$ and separates $K$ from infinity. All the unstable CMC spheres constructed can be parameterized by its mean curvature $H$. If we denote the area of $\Sigma(H)$ by $|\Sigma(H)|$ and $l_{0}=\inf _{x \in \Sigma}|x|$ then we have

$$
\begin{gathered}
l_{0}=O\left(H^{-1}\right), \\
\left|H^{2}\right| \Sigma(H)|-48 \pi| \leq C(p) H .
\end{gathered}
$$


This theorem answers Tian's question partially. In the case of [12] (hence in the case of [7]), the stability condition can not be removed.

In a more recent work [2] of S. Brendle and M. Eichmair, they constructed outlying CMC spheres which are stable in asymptotically Schwarzschild manifold with positive mass. By "outlying" they meant that the compact region bounded by $\Sigma$ is disjoint from $B_{l_{0}(\Sigma)}(0)$. Our approach also works in such a setting.

Theorem 1.2. There is an asymptotically Schwarzschild manifold $(M, g)$ with $m=1$ in which we can construct two sequences of embedded outlying CMC spheres which are parameterized by $\Sigma_{n}^{1}, \Sigma_{n}^{2}$ and a sequence of CMC spheres $\Sigma_{n}^{3}$ which separate the compact part from infinity, such that

$$
H\left(\Sigma_{n}^{1}\right)=H\left(\Sigma_{n}^{2}\right)=H\left(\Sigma_{n}^{3}\right)
$$

and $\Sigma_{n}^{1}, \Sigma_{n}^{3}, \Sigma_{n}^{2}$ share a common symmetry axis and $\Sigma_{n}^{1}, \Sigma_{n}^{2}$ are tangent to $\Sigma_{n}^{3}$ at the two poles of $\Sigma_{n}^{3}$ where $x_{2}=x_{3}=0$.

This result is a little weaker than that of [2] in that we do not discuss the stability of the surfaces.

The main technique of this paper is direct analysis of a nonlinear ODE which comes from the study of Delaunay type CMC surfaces. In a recent work 8], Frank Pacard and I proved that along any non-degenerate closed embedded geodesic in a 3-manifold, one can construct constant mean curvature surfaces of Delaunay type of arbitrarily small size. It was asked by Frank Pacard that if we can deform the neck size of a Delaunay type CMC surface along a closed geodesic to develop a singularity (which can be regarded as the boundary of the moduli of $\mathrm{CMC}$ surfaces). If one can do this and prove that the singularity is removable in certain sense, then one can construct CMC clusters in Riemannian manifolds. In certain sense, this paper is also related to this problem. The unstable CMC spheres constructed are in fact CMC clusters of "three bubbles" in asymptotically Schwarzschild manifold. The author believes that this ODE method can be used to construct many examples. Also we can use it to treat the question raised by Pacard in the case that the metric has rotational symmetry along the geodesic.

The main contributions can be summarized as follows: The meridian curve of the CMC surfaces of revolution is determined by an ODE of the following type

$$
\left\{\begin{array}{l}
g_{a}^{\prime \prime}(y) \quad-\frac{1}{g_{a}(y)}\left(1+g_{a}^{\prime}(y)^{2}\right)+(2+\rho)\left(1+g_{a}^{\prime}(y)^{2}\right)^{\frac{3}{2}}=0 \\
g_{a}(0)=a \in[0.95,1.05] \\
g_{a}^{\prime}(0)=0
\end{array}\right.
$$

where the expression of $\rho=\rho\left(H, g_{a}, g_{a}^{\prime}, y\right)$ is given by (77) and $|\rho| \leq C H$ holds, where $H$ is the mean curvature, which is assumed to be a small positive number. If we neglect $\rho$, when $a<1$, the solution is the meridian curve of Delaunay unduloid in $\mathbb{R}^{3}$. As $a \rightarrow 1^{-}$the solution develops singularities at each integer at the same time. And the Delaunay surface converges to infinitely many spheres each one of which meets its two neighbors at its two poles. If we do not neglect $\rho$, for a fixed $a<1$, when $H$ is very small, the solution looks similar to $\rho=0$ 
case. Look at the graph below. The difference is that the solution is no longer exactly periodic. When $a$ goes up, the solution $g_{a}(y), y \in[0,5]$ will develop a singularity. The singularity will develop at $z_{1}$ or $z_{2}$. To study this, we use the Delaunay parameter function

$$
\tau\left(g_{a}(y), g_{a}^{\prime}(y)\right)=-g_{a}^{2}(y)+\frac{g_{a}(y)}{\sqrt{1+g_{a}^{\prime}(y)^{2}}} .
$$

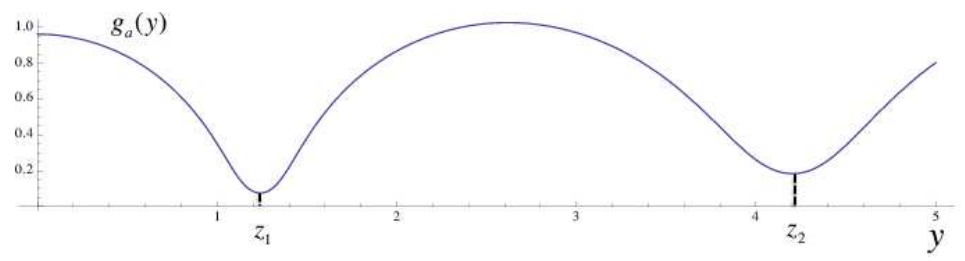

Before a singularity develops, $\tau\left(g_{a}\left(z_{1}\right), g_{a}^{\prime}\left(z_{1}\right)\right), \tau\left(g_{a}\left(z_{2}\right), g_{a}^{\prime}\left(z_{2}\right)\right)>0$. When a singularity develops, $g_{a} \rightarrow 0, g_{a}^{\prime} \rightarrow \infty$, so $\tau \rightarrow 0$. So we will compare $\tau\left(g_{a}\left(z_{1}\right), g_{a}^{\prime}\left(z_{1}\right)\right)$ with $\tau\left(g_{a}\left(z_{2}\right), g_{a}^{\prime}\left(z_{2}\right)\right)$. Note that we have

$$
\frac{d}{d y} \tau\left(g_{a}(y), g_{a}^{\prime}(y)\right)=g_{a} g_{a}^{\prime} \rho .
$$

So

$$
\tau\left(g_{a}\left(z_{2}\right), g_{a}^{\prime}\left(z_{2}\right)\right)-\tau\left(g_{a}\left(z_{1}\right), g_{a}^{\prime}\left(z_{1}\right)\right)=\int_{z_{1}}^{z_{2}} g_{a} g_{a}^{\prime} \rho d y .
$$

So we have to study the function $\rho$. In our case, after some more calculations and arguments, we know (7) is reduced to

$\rho=\frac{H}{d}+\frac{H\left(g_{a}-y g_{a}^{\prime}\right)}{d^{3} \sqrt{1+g_{a}^{\prime 2}}}+\frac{H^{2}(\phi-3)\left(g_{a}-y g_{a}^{\prime}\right)}{4 d^{3} \sqrt{1+g_{a}^{\prime 2}}}-\frac{H^{2} \phi^{\prime}\left(y+g_{a} g_{a}^{\prime}\right)}{8 d^{2} y^{2} \sqrt{1+g_{a}^{\prime 2}}}+C(p, \lambda) O\left(H^{3}\right)$

where $\phi$ is given by (1). Note that $\phi$ has two parameters $\lambda, p$. At first glance, $\phi$ only appears in $H^{2}$ terms, which is not dominant. Here our key observation is that when $H$ is very small

$$
\int_{z_{1}}^{z_{2}} g_{a} g_{a}^{\prime}\left(\frac{H}{d}+\frac{H\left(g_{a}-y g_{a}^{\prime}\right)}{d^{3} \sqrt{1+g_{a}^{\prime 2}}}\right) d y=O\left(H^{2}\right)
$$

which is verified in Lemma 4.7 So when $|p|$ is very large, the third term becomes dominant. So we have freedom to choose the place where the singularity develops, by choosing proper $p$ and $\lambda$. Despite the singularity, the surface of revolution is still smooth from Lemma 4.9. If the singularity develops at $z_{2}$, we can get the "three-bubble" like CMC sphere by applying symmetric extension. Still, we can choose $\lambda$ and $p$ properly such that the singularity develops simultaneously at $z_{1}$ and $z_{2}$. Then we can construct outlying CMC spheres.

The paper is organized as follows. In Section 2, we derive the ODE which is satisfied by the meridian curve of a surface of revolution with constant mean 
curvature. In Section 3, we analyze this ODE and prove the existence of the solution. In Section 4, we deform the Delaunay parameter until the singularity appears. And we can analyze the behavior of the solution at the singular point. In Section 5, we prove the two theorems.

\section{The mean curvature}

Suppose a surface of revolution $\Sigma \subset M$ is defined by

$$
r=f(x)
$$

where $f(x)$ is a smooth positive function. We are going to calculate the mean curvature of such a surface.

We assume $(s, \theta)$ to be the coordinate on the surface which satisfies

$$
\frac{\partial}{\partial s}=\frac{\partial}{\partial x}+\frac{\partial f}{\partial x} \frac{\partial}{\partial r}, \frac{\partial}{\partial \theta}=x_{2} \frac{\partial}{\partial x_{3}}-x_{3} \frac{\partial}{\partial x_{2}} .
$$

We have

$$
\left\{\begin{array}{l}
g\left(\partial_{s}, \partial_{s}\right)=1+f^{\prime}(x)^{2}+g_{3}+f^{\prime}(x)^{2} g_{1}, \\
g\left(\partial_{\theta}, \partial_{\theta}\right)=r^{2}+g_{2} .
\end{array}\right.
$$

The unit normal vector on $\Sigma$ is

$$
V=V_{r} \partial_{r}+V_{x} \partial_{x}
$$

where

$$
\left\{\begin{array}{l}
V_{r}=\frac{1}{\sqrt{1+g_{1}} \sqrt{1+\frac{1+g_{1}}{1+g_{3}} f^{\prime 2}}}, \\
V_{x}=-f^{\prime} \frac{\sqrt{1+g_{1}}}{\left(1+g_{3}\right) \sqrt{1+\frac{1+g_{1}}{1+g_{3}} f^{\prime 2}}} .
\end{array}\right.
$$

We use $V_{e}$ to represent the normal vector in Euclidean metric, i.e.

$$
V_{e}=\frac{\partial_{r}-f^{\prime} \partial_{x}}{\sqrt{1+f^{\prime 2}}}
$$

We use $<\cdot, \cdot>$ to represent the inner product in the metric $g$ and "." to represent the inner product in the Euclidean metric. The mean curvature

$$
\begin{gathered}
H(\Sigma)=g^{s s}<\nabla_{\partial_{s}} V, \partial_{s}>+g^{\theta \theta}<\nabla_{\partial_{\theta}} V, \partial_{\theta}> \\
<\nabla_{\partial_{s}} V, \partial_{s}>= \\
<\left(\partial_{s} V_{r}\right) \partial_{r}, \partial_{s}>+<V_{r} \nabla_{\partial_{s}} \partial_{r}, \partial_{s}> \\
+<\left(\partial_{s} V_{x}\right) \partial_{x}, \partial_{s}>+<V_{x} \nabla_{\partial_{s}} \partial_{x}, \partial_{s}>.
\end{gathered}
$$


By direct calculations we have

$$
\begin{aligned}
<\left(\partial_{s} V_{r}\right) \partial_{r}, \partial_{s}> & =\left(\partial_{s} V_{r}\right) f^{\prime}\left(1+g_{1}\right), \\
<\left(\partial_{s} V_{x}\right) \partial_{x}, \partial_{s}> & =\left(\partial_{s} V_{x}\right)\left(1+g_{3}\right), \\
<V_{r} \nabla_{\partial_{s}} \partial_{r}, \partial_{s}> & =\frac{V_{r}}{2}\left(\partial_{r} g_{x x}+f^{\prime 2} \partial_{r} g_{r r}\right), \\
<V_{x} \nabla_{\partial_{s}} \partial_{x}, \partial_{s}> & =\frac{V_{x}}{2}\left(\partial_{x} g_{x x}+f^{\prime 2} \partial_{x} g_{r r}\right), \\
<\nabla_{\partial_{\theta}} V, \partial_{\theta}> & =V_{r}<\nabla_{\partial_{\theta}} \partial_{r}, \partial_{\theta}>+V_{x}<\nabla_{\partial_{\theta}} \partial_{x}, \partial_{\theta}> \\
= & \frac{1}{2}\left(V_{r} \partial_{r} g_{\theta \theta}+V_{x} \partial_{x} g_{\theta \theta}\right) .
\end{aligned}
$$

So we get

$$
\begin{aligned}
H(\Sigma) & =\left(1+f^{\prime}(x)^{2}+g_{3}+f^{\prime}(x)^{2} g_{1}\right)^{-1}\left[\left(\partial_{s} V_{r}\right) f^{\prime}\left(1+g_{1}\right)+\left(\partial_{s} V_{x}\right)\left(1+g_{3}\right)\right. \\
& \left.+\frac{V_{r}}{2}\left(\partial_{r} g_{x x}+f^{\prime 2} \partial_{r} g_{r r}\right)+\frac{V_{x}}{2}\left(\partial_{x} g_{x x}+f^{\prime 2} \partial_{x} g_{r r}\right)\right] \\
& +\left(r^{2}+g_{2}\right)^{-1} \frac{1}{2}\left(V_{r} \partial_{r} g_{\theta \theta}+V_{x} \partial_{x} g_{\theta \theta}\right) .
\end{aligned}
$$

From (3), the expression of $H(\Sigma)$ can be reduced to

$$
\begin{aligned}
H(\Sigma)= & \frac{1}{2}\left(1+g_{1}\right)^{-\frac{3}{2}}\left(1+f^{\prime 2}\right)^{-\frac{3}{2}}\left(-2\left(1+g_{1}\right) f^{\prime \prime}\right. \\
& +\left(1+f^{\prime 2}\right)\left(\partial_{r} g_{1}-f^{\prime} \partial_{x} g_{1}\right) \\
& \left.+\left(r^{2}+g_{2}\right)^{-1}\left(1+g_{1}\right)\left(1+f^{\prime 2}\right)\left(2 r+\partial_{r} g_{2}-f^{\prime} \partial_{x} g_{2}\right)\right) \\
= & -\left(1+g_{1}\right)^{-\frac{1}{2}}\left(1+f^{\prime 2}\right)^{-\frac{3}{2}} f^{\prime \prime}-2\left(V_{e} \cdot x^{\prime}\right)\left(l^{-3}+l^{-4}\right)\left(1+g_{1}\right)^{-\frac{3}{2}} \\
& +\left(1+g_{1}\right)^{-\frac{1}{2}}\left(1+f^{\prime 2}\right)^{-\frac{1}{2}} f^{-1}+\frac{1}{2}\left(1+g_{1}\right)^{-\frac{1}{2}} \chi(r, x)^{-1} V_{e}(\chi) .
\end{aligned}
$$

where $x^{\prime}=(x, f(x) \cos \theta, f(x) \sin \theta)$ and $V_{e}(\chi)$ is the derivative of $\chi(r, x)$ in the direction $V_{e}=\frac{\partial_{r}-f^{\prime} \partial_{x}}{\sqrt{1+f^{\prime 2}}}$.

Now we have

$$
\frac{d^{2} f}{d x^{2}}-\frac{1}{f}\left(1+\left(\frac{d f}{d x}\right)^{2}\right)+(H+\tilde{\rho})\left(1+\left(\frac{d f}{d x}\right)^{2}\right)^{\frac{3}{2}}=0,
$$

where

$$
\tilde{\rho}=\frac{H}{l}+2\left(V_{e} \cdot x^{\prime}\right)\left(l^{-3}+l^{-4}\right)\left(1+g_{1}\right)^{-1}-\frac{1}{2} \chi^{-1} V_{e}(\chi) .
$$

Since we want to get constant mean curvature surfaces, we assume $H$ to be a small positive constant. Denote

$$
g(y)=\frac{H}{2} f\left(\frac{2}{H} y\right)=\frac{H}{2} f(x) .
$$


We have

$$
g^{\prime}(y)=f_{x}\left(\frac{2}{H} y\right), g^{\prime \prime}(y)=\frac{2}{H} f_{x x}\left(\frac{2}{H} y\right),
$$

So we have

$$
g^{\prime \prime}(y)-\frac{1}{g(y)}\left(1+g^{\prime}(y)^{2}\right)+(2+\rho)\left(1+g^{\prime}(y)^{2}\right)^{\frac{3}{2}}=0
$$

where

$$
\rho=\frac{2}{H} \tilde{\rho} .
$$

We want to study ODE (5) instead of (4).

\section{The analysis of the ODE}

Consider

$$
\left\{\begin{array}{l}
g^{\prime \prime}(y)-\frac{1}{g(y)}\left(1+g^{\prime}(y)^{2}\right)+(2+\rho)\left(1+g^{\prime}(y)^{2}\right)^{\frac{3}{2}}=0, \\
g(0)=a \\
g^{\prime}(0)=0 .
\end{array}\right.
$$

We assume $a \in[0.9,1.1]$ and denote the solution by $g_{a}(y)$. Let us assume that $H>0$ is very small and the real number $p$ is fixed. Let $d=\frac{H}{2} l$. Then we have

$$
\begin{aligned}
\rho= & \frac{H}{d}+\left(\frac{H}{2}+\frac{H^{2}}{4 d}\right) \frac{g_{a}-y g_{a}^{\prime}}{d^{3}\left(1+g_{1}\right) \sqrt{1+g_{a}^{\prime 2}}} \\
& +\left(\frac{H}{2}+\frac{H^{2} \phi}{4 d}\right) \frac{g_{a}-y g_{a}^{\prime}}{d^{3}\left(1+\frac{H}{d}+\frac{\phi H^{2}}{4 d^{2}}\right) \sqrt{1+g_{a}^{\prime 2}}} \\
& -\frac{H^{2}}{8 d^{2}} \frac{\phi^{\prime}}{\left(1+\frac{H}{d}+\frac{\phi H^{2}}{4 d^{2}}\right)} \frac{y+g_{a} g_{a}^{\prime}}{y^{2} \sqrt{1+g_{a}^{\prime 2}}} .
\end{aligned}
$$

In the following analysis, by a constant $C$ ( or $C_{i}, i=1,2, \cdots$ ) we mean a general (or a particular) uniform positive constant which does not depend on $H$, $a, p, \lambda$ or $y$. We use symbols $C(H), C(p)$ to denote constants which depend on $H, p$, etc.

First we have the local existence. Let $A_{1}, A_{2}, A_{3}, A_{4}$ be four positive constant. Let's denote a domain in the phase space

$$
\left\{\left(g, g^{\prime}\right) ; A_{2} \leq g \leq A_{1},-A_{4} \leq g^{\prime} \leq A_{3}\right\}
$$

as $D\left(A_{1}, A_{2}, A_{3}, A_{4}\right)$.

Lemma 3.1. Suppose $g_{a}(y), y \in[\alpha, \beta], 0 \leq \alpha \leq \beta$ solves (6). And for some $A_{i}, i=1, \cdots, 4,\left(g_{a}, g_{a}^{\prime}\right) \in D\left(A_{1}, A_{2}, A_{3}, A_{4}\right), y \in[\alpha, \beta]$. Then there is $\delta>0$ such that the solution $g_{a}$ can be extended to $[\alpha, \beta+\delta)$. 
Proof. Consider a system that is equivalent to (6)

$$
\left\{\begin{array}{l}
g_{a}^{\prime}=w_{a} \\
w_{a}^{\prime}=\frac{1}{g_{a}}\left(1+w_{a}^{2}\right)-(2+\rho)\left(1+w_{a}^{2}\right)^{\frac{3}{2}} .
\end{array}\right.
$$

When $\left(g_{a}, w_{a}\right) \in D\left(A_{1}, A_{2}, A_{3}, A_{4}\right)$ and $y \in[\alpha, \beta]$, we know $d$ has lower bound $A_{2}$. The right hand side has uniform bound and uniform Lipschitz constant with respect to $g_{a}, w_{a}$. From the local existence of ODE system, we can prove this lemma.

Then we have a "closed" property for the solution.

Lemma 3.2. Suppose $g_{a}(y), y \in[\alpha, \beta), 0 \leq \alpha<\beta<+\infty$ solves (6)). And for some $A_{i}>0, i=1, \cdots, 4,\left(g_{a}, g_{a}^{\prime}\right) \in D\left(A_{1}, A_{2}, A_{3}, A_{4}\right), y \in[\alpha, \beta)$. If

$$
\lim _{y \rightarrow \beta^{-}} g_{a}(y), \lim _{y \rightarrow \beta^{-}} g_{a}^{\prime}(y)
$$

exist and are finite, then $g_{a}(y)$ (as a solution to (6)) can be extended to $y=\beta$. In particular, if $g_{a}(y)$ and $g_{a}^{\prime}(y)$ are both monotonic and bounded, $g_{a}$ can be extended to $y=\beta$.

Proof. If for some $A_{i}, i=1, \cdots, 4,\left(g_{a}, g_{a}^{\prime}\right) \in D\left(A_{1}, A_{2}, A_{3}, A_{4}\right), y \in[\alpha, \beta)$ and

$$
\lim _{y \rightarrow \beta^{-}} g_{a}(y), \lim _{y \rightarrow \beta^{-}} g_{a}^{\prime}(y)
$$

exist, then from (6) and (7) we have

$$
\lim _{y \rightarrow \beta^{-}} g_{a}^{\prime \prime}(y)
$$

exists. By defining the values of $g_{a}, g_{a}^{\prime}, g_{a}^{\prime \prime}$ on $\beta$ in the obvious way, we can prove this lemma.

Lemma 3.3. Suppose $g_{a}(y)>0, y \in I$ solves (6) where $I$ is an interval $[\alpha, \beta]$ or $[\alpha, \beta), 0 \leq \alpha<\beta$. In the case of $I=[\alpha, \beta), g_{a}(\beta)$ and $g_{a}^{\prime}(\beta)$ should be understood as a limit which is assumed to exist.

Then

1. If $g_{a}(y)$ is monotonic in $y$ and $0<C^{-1}<\left|g_{a}^{\prime}\right|<C$ when $y \in I$, then

$$
C^{-1}\left|g_{a}(\beta)-g_{a}(\alpha)\right| \leq|\beta-\alpha| \leq C\left|g_{a}(\beta)-g_{a}(\alpha)\right| \text {. }
$$

2. If $g_{a}^{\prime}(y)$ is monotonic in $y$ and $k_{a}=g_{a} \sqrt{1+g_{a}^{\prime}(y)^{2}}$ satisfies that $k_{a}-\frac{1}{2}$ does not change sign and

$$
C^{-1} \leq\left|k_{a}-\frac{1}{2}\right| \leq C, y \in I
$$


And we assume that $|\rho| \leq C H$. Then when $H$ is small we have

$$
C^{-1} \inf _{y \in I} g_{a} \leq \frac{|\beta-\alpha|}{\left|\arctan g_{a}^{\prime}(\beta)-\arctan g_{a}^{\prime}(\alpha)\right|} \leq C \sup _{y \in I} g_{a} .
$$

Proof. We need only prove the case $I=[\alpha, \beta]$. The case $I=[\alpha, \beta)$ follows by considering $\left[\alpha, \beta_{i}\right]$ with $\beta_{i} \rightarrow \beta$.

The first one follows form

$$
|\beta-\alpha|=\left|\int_{g_{a}(\alpha)}^{g_{a}(\beta)} \frac{d g_{a}}{g_{a}^{\prime}}\right|
$$

and the second one follows from the fact that $\rho$ is small and

$$
|\beta-\alpha|=\left|\int_{g_{a}^{\prime}(\alpha)}^{g_{a}^{\prime}(\beta)} \frac{d g_{a}^{\prime}}{g_{a}^{\prime \prime}}\right|=\left|\int_{g_{a}^{\prime}(\alpha)}^{g_{a}^{\prime}(\beta)} \frac{g_{a} d g_{a}^{\prime}}{\left(1+g_{a}^{\prime 2}\right)\left(1-(2+\rho) k_{a}\right)}\right| .
$$

Now we will prove that for the solution of (6),$d=\sqrt{y^{2}+g_{a}^{2}}$ has uniform positive lower bound independent of $H$ and $a \in[0,9,1,1]$. This is an important estimate. Only with this estimate can we know that $|\rho| \leq C H$.

Lemma 3.4. We can choose $\lambda>0$ small and $\delta(p, \lambda)>0$ such that when $H<$ $\delta(p, \lambda)$, for all $a \in[0.9,1.1]$, as long as the solution of (6) can be extended when $y>0$,

$$
\begin{gathered}
d=\sqrt{g_{a}^{2}+y^{2}} \geq C>0 \\
\left|\frac{1}{8 d^{2}} \frac{\phi^{\prime}}{\left(1+\frac{H}{d}+\frac{\phi H^{2}}{4 d^{2}}\right)} \frac{y+g g^{\prime}}{y^{2} \sqrt{1+g_{a}^{\prime 2}}}\right| \leq \frac{C|p-1|}{\lambda}, \\
|\rho| \leq \hat{C} H .
\end{gathered}
$$

Proof. First let us pretend that $\phi=p$ holds everywhere. So $\phi^{\prime}=0$ and

$$
\begin{aligned}
\rho= & \frac{H}{d}+\frac{H}{2} \frac{g_{a}-y g_{a}^{\prime}}{d^{3}\left(1+g_{1}\right) \sqrt{1+g_{a}^{\prime 2}}}+\frac{H^{2}}{4} \frac{g_{a}-y g_{a}^{\prime}}{d^{4}\left(1+g_{1}\right) \sqrt{1+g_{a}^{\prime 2}}} \\
& +\frac{H}{2} \frac{g_{a}-y g_{a}^{\prime}}{d^{3}\left(1+\frac{H}{d}+\frac{\phi H^{2}}{4 d^{2}}\right) \sqrt{1+g_{a}^{\prime 2}}}+\frac{H^{2}}{4} \frac{p\left(g_{a}-y g_{a}^{\prime}\right)}{d^{4}\left(1+\frac{H}{d}+\frac{\phi H^{2}}{4 d^{2}}\right) \sqrt{1+g_{a}^{\prime 2}}} .
\end{aligned}
$$

Let $D_{a}(y)$ solves

$$
\left\{\begin{array}{l}
D^{\prime \prime}(y)-\frac{1}{D(y)}\left(1+D^{\prime}(y)^{2}\right)+2\left(1+D^{\prime}(y)^{2}\right)^{\frac{3}{2}}=0 \\
D(0)=a \\
D^{\prime}(0)=0
\end{array}\right.
$$

where $a \in[0.8,1.2]$.

We state some facts of $D_{a}(y)$ without proof. There are $\delta_{0}, C_{1}, C_{2}, C_{3}>0$ such that, 
1. For each $a \in[0.8,1.2]$, the solution of (9) exists on $\left[0, \delta_{0}\right]$.

2. $R_{1}=\left\{\left(D_{a}(y), \frac{d}{d y} D_{a}(y)\right) ; a \in[0.8,1.2], y \in\left[0, \delta_{0}\right]\right\}$ is trapped in the region

$$
R_{2}=\left\{(\xi, \eta) ; C_{1} \leq \xi \leq 1.2,-C_{2} \leq \eta \leq 0\right\} .
$$

3. When $a \in[0.8,1.2]$, the solution $D(y), y \in\left[0, \delta_{0}\right]$ satisfies $D \sqrt{1+D^{\prime 2}} \geq \frac{2}{3}$.

4. For each $a \in[0.8,1.2], D_{a}(0)-D_{a}\left(\delta_{0}\right)>C_{3}$ and $D_{a}^{\prime}\left(\delta_{0}\right)<-C_{3}$.

We may draw a graph in the phase space to illustrate this. In the graph below, $\delta_{0}=0.75$. We can choose $C_{1}=0.5, C_{2}=2.4 . D_{a}(0)-D_{a}\left(\delta_{0}\right)$ and $-D_{a}^{\prime}\left(\delta_{0}\right)$ are positive continuous functions of $a \in[0,8,1.2]$. So $C_{3}$ can be chosen properly small and positive.

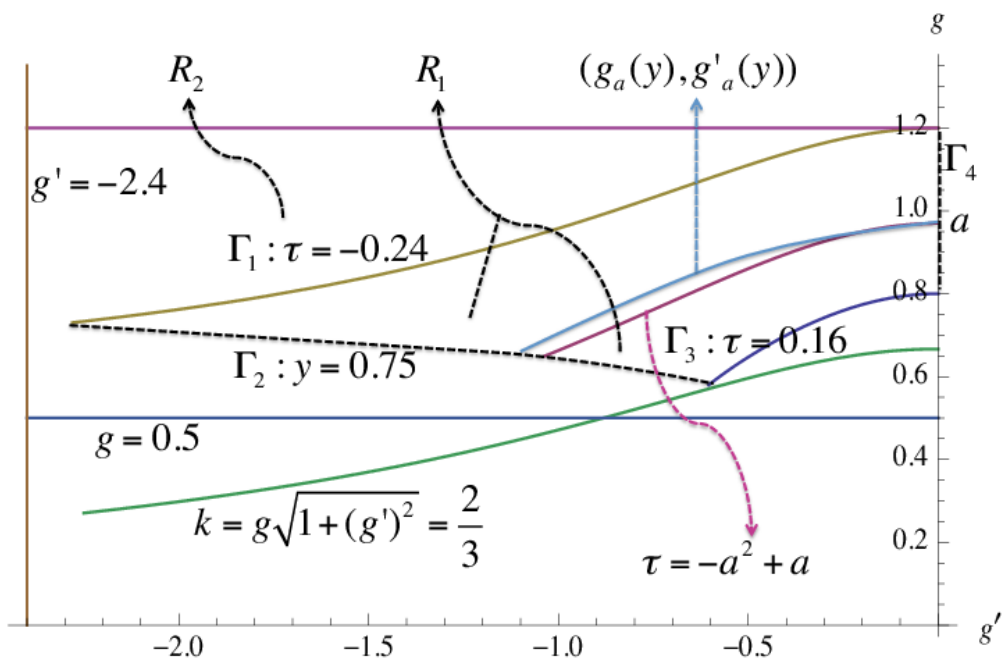

Let $R_{1}$ be the closure of a bounded domain whose boundary contains 4 components, $\Gamma_{1}, \Gamma_{2}, \Gamma_{3}, \Gamma_{4}$.

$$
\begin{aligned}
& \Gamma_{1}=\left\{\left(D_{a}(y), \frac{d}{d y} D_{a}(y)\right) ; a=1.2, y \in\left[0, \delta_{0}\right]\right\}, \\
& \Gamma_{2}=\left\{\left(D_{a}(y), \frac{d}{d y} D_{a}(y)\right) ; a \in[0.8,1.2], y=\delta_{0}\right\}, \\
& \Gamma_{3}=\left\{\left(D_{a}(y), \frac{d}{d y} D_{a}(y)\right) ; a=0.8, y \in\left[0, \delta_{0}\right]\right\}, \\
& \Gamma_{4}=\left\{\left(D_{a}(y), \frac{d}{d y} D_{a}(y)\right) ; a \in[0.8,1.2], y=0\right\} .
\end{aligned}
$$

Now we consider the orbit $\left(g_{a}, g_{a}^{\prime}\right)$ of (6) . For each $a \in[0,9,1.1],\left(g_{a}(0), g_{a}^{\prime}(0)\right) \in$ $\Gamma_{4}$. From Lemma 3.1, the solution of (6) can be extended a little to $y>0$. Then 
the orbit will be extended into the interior of $R_{1}$. As long as the orbit keeps in $R_{1}$, we know $d \geq C_{1}$. So there are $\delta(p)>0, C>0$ such that when $0<H<\delta(p)$,

$$
|\rho| \leq C H \text {. }
$$

If $\left\{\left(g_{a}(y), g_{a}^{\prime}(y)\right) ; y \in\left[0, \delta_{0}\right]\right\} \subset R_{1}$, we have $d=\sqrt{y^{2}+d^{2}} \geq \min \left\{\delta_{0}, C_{1}\right\}$ as long as the solution exists. If it is not the case then we assume $y^{\prime} \in\left(0, \delta_{0}\right)$ is the first time $\left(g_{a}, g_{a}^{\prime}\right)$ touches $\partial R_{1}$. We claim that for $H$ sufficiently small, $\left(g_{a}\left(y^{\prime}\right), g_{a}^{\prime}\left(y^{\prime}\right)\right) \in \Gamma_{2}$. For this we consider the Delaunay parameter function

$$
\tau\left(g_{a}, g_{a}^{\prime}\right)=-g_{a}^{2}+\frac{g_{a}}{\sqrt{1+g_{a}^{\prime 2}}} .
$$

One can regard it as the first integral of (9). When $y \in\left[0, y^{\prime}\right]$

$$
\frac{d}{d y} \tau=g_{a} g_{a}^{\prime} \rho \text {. }
$$

Note that $\left|g_{a} g_{a}^{\prime}\right| \leq 1.2 C_{2}$. From (10), by choosing $H$ even smaller, we have for each $a \in[0,9,1.1]$, as long as $\left(g_{a}, g_{a}^{\prime}\right) \in R_{1},\left(g_{a}, g_{a}^{\prime}\right)$ keeps in a small neighborhood of $\left(D_{a}, D_{a}^{\prime}\right)$. So $\left(g_{a}, g_{a}^{\prime}\right)$ has no chance to touch $\Gamma_{1}$ or $\Gamma_{3}$. From

$$
g_{a} \sqrt{1+g_{a}^{\prime 2}} \geq \frac{2}{3}
$$

and the fact that $\rho$ is small, we can deduce

$$
g_{a}^{\prime \prime}(y)=\frac{1}{g_{a}(y)}\left(1+g_{a}^{\prime}(y)^{2}\right)\left(1-(2+\rho) g_{a}(y)\left(1+g_{a}^{\prime}(y)^{2}\right)^{\frac{1}{2}}\right)
$$

is always negative, which implies both $g_{a}$ and $g_{a}^{\prime}$ are monotonically decreasing when $y \in\left[0, y^{\prime}\right]$. From Lemma 3.3, we have

$$
C\left(C_{1}, C_{2}, C_{3}\right)^{-1} \leq y^{\prime} \leq C\left(C_{1}, C_{2}, C_{3}\right) .
$$

So if we choose $2 \lambda \leq C\left(C_{1}, C_{2}, C_{3}\right)^{-1} C_{1}$ and define $\phi$ as (1), it will have no influence on the analysis above. And we know

$$
d \geq \min \left\{C\left(C_{1}, C_{2}, C_{3}\right)^{-1}, C_{1}, \delta_{0}\right\} .
$$

Note that when $\phi^{\prime} \neq 0$, there hold $y \geq C\left(C_{1}, C_{2}, C_{3}\right)^{-1}$ and $\left|\frac{g}{y}\right| \leq 2 \lambda$. So from (2)

$$
\begin{aligned}
& \left|\frac{1}{8 d^{2}} \frac{\phi^{\prime}}{\left(1+\frac{H}{d}+\frac{\phi H^{2}}{4 d^{2}}\right)}\left(1+g^{\prime 2}\right)^{-\frac{1}{2}} \frac{y+g g^{\prime}}{y^{2}}\right| \\
\leq & \frac{C|p-1|}{\lambda} .
\end{aligned}
$$

So from

$$
\frac{\left|g_{a}-y g_{a}^{\prime}\right|}{\sqrt{1+g_{a}^{\prime 2}} \sqrt{y^{2}+g_{a}^{2}}} \leq 1
$$

we can choose $\delta(p, \lambda)$ small such that $|\rho| \leq \hat{C} H$. 
Now we can expand $\rho$ in terms of $H$. For $0<H<\delta(p, \lambda)$,

$\rho=\frac{H}{d}+\frac{H\left(g_{a}-y g_{a}^{\prime}\right)}{d^{3} \sqrt{1+g_{a}^{\prime 2}}}+\frac{H^{2}(\phi-3)\left(g_{a}-y g_{a}^{\prime}\right)}{4 d^{3} \sqrt{1+g_{a}^{\prime 2}}}-\frac{H^{2} \phi^{\prime}\left(y+g_{a} g_{a}^{\prime}\right)}{8 d^{2} y^{2} \sqrt{1+g_{a}^{\prime 2}}}+C(p, \lambda) O\left(H^{3}\right)$.

From now on we will always assume that $\delta(p, \lambda)$ is so small that $|\rho| \leq \hat{C} H$.

It is obviously that exactly one of the following three will happen to $g_{a}(y)$,

(H1): The solution $g_{a}(y)$ can be extended at least two times such that $g_{a}(y) \in$ $\left(0, \frac{1}{2}\right), g_{a}^{\prime}(y)=0$

(H2): The solution $g_{a}(y)$ can be extended only one time such that $g_{a}(y) \in$ $\left(0, \frac{1}{2}\right), g_{a}^{\prime}(y)=0$

(H3): The solution $g_{a}(y)$ cannot be extended to a point $y$ such that $g_{a}(y) \in$ $\left(0, \frac{1}{2}\right), g_{a}^{\prime}(y)=0$.

Let's draw some pictures of the phase space to illustrate the three cases.

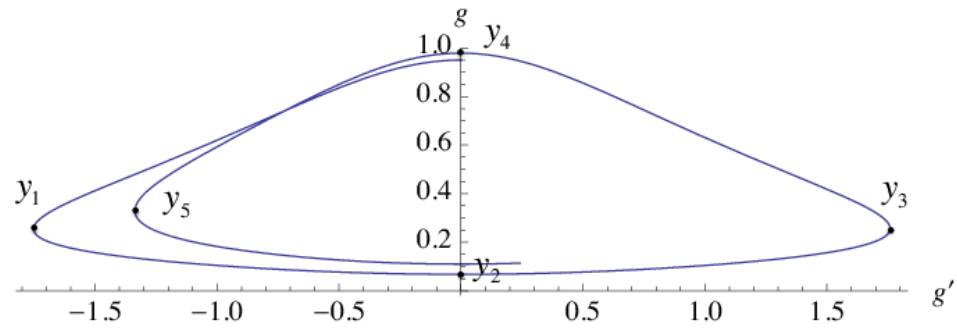

H1 happens

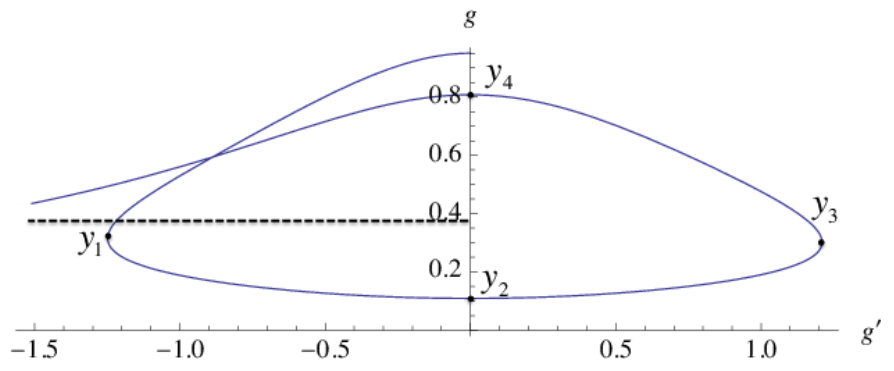

H2 happens

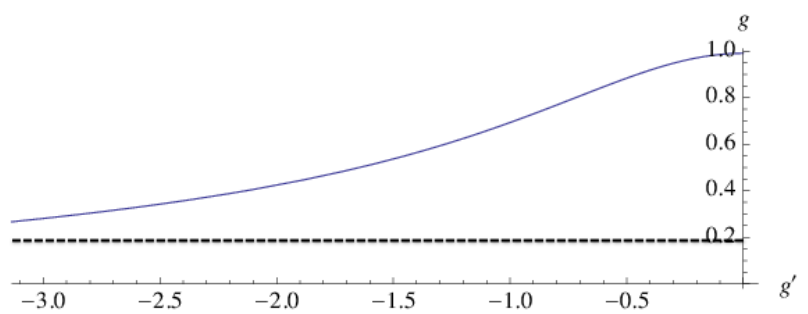

H3 happens 
Lemma 3.5. There is a uniform $C_{4}>0$ such that if $g_{a}(y)$ can be extended to some $y_{1}>0$ such that $0<g_{a}\left(y_{1}\right)<C_{4}, g_{a}^{\prime}\left(y_{1}\right)<0$ and $g_{a}^{\prime \prime}\left(y_{1}\right)=0$, then there is $y_{2}>y_{1}$ such that $g_{a}\left(y_{2}\right) \in\left(0, \frac{1}{2}\right)$ and $g_{a}^{\prime}\left(y_{2}\right)=0$. Moreover, in $\left[y_{1}, y_{2}\right], g_{a}(y)$ is monotonically decreasing and $g_{a}^{\prime}(y)$ is monotonically increasing. (This can be seen in the case "H1" and "H2".)

Proof. From Lemma 3.1, we know that the solution can be extended beyond $y_{1}$ for a small interval. We want to show that, when $y>y_{1}$, as long as the solution exists and $g_{a}>0$ and $g_{a}^{\prime}<0$, we have $g_{a}^{\prime \prime}>0$. As before we denote

$$
k_{a}(y)=g_{a} \sqrt{1+g_{a}^{\prime 2}} .
$$

Note that $|\rho| \leq \hat{C} H$. If $k_{a}(y)<\frac{1}{2+\hat{C} H}$, we have

$$
g_{a}^{\prime \prime}(y)=\frac{1}{g_{a}}\left(1+g_{a}^{\prime 2}\right)\left(1-(2+\rho) k_{a}\right)>0 .
$$

From easy calculation

$$
\frac{d k_{a}(y)}{d y}=g_{a}^{\prime}\left(1+g_{a}^{\prime 2}\right)^{\frac{1}{2}}+g_{a}\left(1+g_{a}^{\prime 2}\right)^{-\frac{1}{2}} g_{a}^{\prime} g_{a}^{\prime \prime} .
$$

When $g_{a}>0, g_{a}^{\prime}<0$, we have

$$
\begin{aligned}
\frac{d k_{a}}{d g_{a}} & =\frac{d k_{a}(y)}{d y} \frac{1}{g_{a}^{\prime}}=\left(1+g_{a}^{\prime 2}\right)^{\frac{1}{2}}+g_{a}\left(1+g_{a}^{\prime 2}\right)^{-\frac{1}{2}} g_{a}^{\prime \prime} \\
& =\frac{k_{a}\left(2-(2+\rho) k_{a}\right)}{g_{a}} .
\end{aligned}
$$

So we have

$$
\frac{d k_{a}}{k_{a}\left(2-(2+\rho) k_{a}\right)}=\frac{d g_{a}}{g_{a}} .
$$

When $y=y_{1}$, from $g_{a}^{\prime \prime}\left(y_{1}\right)=0$, we have $2-\left(2+\rho\left(y_{1}\right)\right) k_{a}\left(y_{1}\right)=1$. So as long as the solution can be extended when $y>y_{1}$ and $g_{a}>0, g_{a}^{\prime}<0$ we have $k_{a}$ is monotonically decreasing. So once $k_{a}(y)<\frac{1}{2+\hat{C} H}, g_{a}^{\prime \prime}(y)>0$ holds as long as $y>y_{1}, g_{a}>0, g_{a}^{\prime}<0$.

The problem is whether we still have $g_{a}^{\prime \prime}(y)>0$, when $y>y_{1}$ and $\frac{1}{2+\rho\left(y_{1}\right)} \geq$ $k_{a} \geq \frac{1}{2+\hat{C} H}$. We choose $0<C_{4}<\frac{1}{\sqrt{2}(2+\hat{C} H)}$. When $0<g_{a}(y) \leq g_{a}\left(y_{1}\right) \leq C_{4}$, $k_{a}=g_{a}(y) \sqrt{1+g_{a}^{\prime 2}(y)} \geq \frac{1}{2+\hat{C} H}$ and $g_{a}^{\prime}(y)<0$ we have

$$
\sqrt{1+g_{a}^{\prime 2}(y)} \geq \sqrt{2}
$$

which implies $g_{a}^{\prime}(y) \leq-1$.

From direct calculations, $\exists$ a uniform constant $C>0$, such that when $H$ is sufficiently small

$$
\begin{aligned}
\left|\rho^{\prime}\right| & \leq\left(\frac{H}{d^{3}} y+\frac{H}{d^{3}} g_{a}\left|g_{a}^{\prime}\right|\right)\left(1+\frac{\left|g_{a}^{\prime \prime}\right|}{\left(1+\left(g_{a}^{\prime}\right)^{2}\right)^{\frac{3}{2}}}\right)+C(p, \lambda) H^{2}\left(1+\left|g_{a}^{\prime}\right|\right) \\
& \leq C H\left(1+\left|g_{a}^{\prime}\right|\right) .
\end{aligned}
$$


From

$$
\frac{d}{d y}\left((2+\rho) k_{a}\right)=\rho^{\prime} k_{a}+(2+\rho) k_{a}^{\prime}
$$

and (14) (16) we have

$$
\frac{d}{d g_{a}}\left((2+\rho) k_{a}\right) \geq \frac{1}{2} \frac{k_{a}}{g_{a}}\left(2+\rho-\left|\frac{2 C H\left(1+g_{a}^{\prime}\right) g_{a}}{g_{a}^{\prime}}\right|\right) .
$$

When $y>y_{1}, k_{a} \geq \frac{1}{2+\hat{C} H}$, from

$$
g_{a}^{\prime} \leq-1
$$

we have, for $H$ sufficiently small, $\frac{d}{d g_{a}}\left((2+\rho) k_{a}\right)$ is positive. (Here we need the estimate $g_{a}^{\prime} \leq-1$. We use $C_{4}$ for this technical reason.) So when $y>y_{1}$ and $\frac{1}{2+\rho\left(y_{1}\right)} \geq k_{a} \geq \frac{1}{2+\hat{C} H}$, we have $(2+\rho) k_{a}$ monotonically decreasing as $y$ increases. So when $y>y_{1}$,

$$
g_{a}^{\prime \prime}(y)=\frac{1}{g_{a}}\left(1+g_{a}^{\prime 2}\right)\left(1-(2+\rho) k_{a}\right)>0 .
$$

By now we have known, when $y>y_{1}$, as long as the solution exists and $0<g_{a} \leq C_{4}$ and $g_{a}^{\prime}<0, g_{a}$ is monotonically decreasing and $g_{a}^{\prime}$ is monotonically increasing. Now we prove that there is a positive lower bound for $g_{a}$. We assume the solution can be extended from $y_{1}$ to $\tilde{y}_{2}$ with $g_{a}\left(\tilde{y}_{2}\right)>0$ and $g_{a}^{\prime}\left(\tilde{y}_{2}\right)<0$. From (15) we know when $y \in\left[y_{1}, \tilde{y}_{2}\right]$

$$
\int_{g_{a}(y)}^{g_{a}\left(y_{1}\right)} \frac{d g_{a}}{g_{a}}=\int_{k_{a}(y)}^{k_{a}\left(y_{1}\right)} \frac{d k_{a}}{k_{a}\left(2-(2+\rho) k_{a}\right)} \leq \int_{k_{a}(y)}^{k_{a}\left(y_{1}\right)} \frac{d k_{a}}{k_{a}\left(2-(2+\hat{C} H) k_{a}\right)} .
$$

So we have

$$
\ln \frac{g_{a}\left(y_{1}\right)}{g_{a}(y)} \leq\left.\left(\frac{1}{2} \ln \frac{(2+\hat{C} H) k_{a}}{2-(2+\hat{C} H) k_{a}}\right)\right|_{k_{a}(y)} ^{k_{a}\left(y_{1}\right)},
$$

which implies

$$
k_{a}(y) \leq C\left(\frac{g_{a}(y)}{g_{a}\left(y_{1}\right)}\right)^{2} .
$$

From

$$
\frac{d g_{a}^{\prime}}{d g_{a}}=\frac{g_{a}^{\prime \prime}}{g_{a}^{\prime}}=\frac{\left(1+\left(g_{a}^{\prime}\right)^{2}\right)\left[1-(2+\rho) k_{a}\right]}{g_{a} g_{a}^{\prime}}
$$

we have

$$
\begin{aligned}
\int_{g_{a}^{\prime}\left(\tilde{y}_{2}\right)}^{g_{a}^{\prime}\left(y_{1}\right)} \frac{g_{a}^{\prime} d g_{a}^{\prime}}{1+\left(g_{a}^{\prime}\right)^{2}} & =\int_{g_{a}\left(\tilde{y}_{2}\right)}^{g_{a}\left(y_{1}\right)} \frac{1-(2+\rho) k_{a}}{g_{a}} d g_{a} \\
& \geq \int_{g_{a}\left(\tilde{y}_{2}\right)}^{g_{a}\left(y_{1}\right)} \frac{1-C\left(\frac{g_{a}(y)}{g_{a}\left(y_{1}\right)}\right)^{2}}{g_{a}(y)} d g_{a}(y)
\end{aligned}
$$


which implies

$$
\left.\frac{1}{2} \ln \left(1+g_{a}^{\prime 2}\right)\right|_{g_{a}^{\prime}\left(\tilde{y}_{2}\right)} ^{g^{\prime}\left(y_{1}\right)} \geq \ln \frac{g_{a}\left(y_{1}\right)}{g_{a}\left(\tilde{y}_{2}\right)}-\frac{C}{2} g_{a}\left(y_{1}\right)^{-2}\left(g_{a}\left(y_{1}\right)^{2}-g_{a}\left(\tilde{y}_{2}\right)^{2}\right) .
$$

So we have

$$
g_{a}\left(\tilde{y}_{2}\right) \geq \mathrm{e}^{-C}\left(1+g_{a}^{\prime}\left(y_{1}\right)^{2}\right)^{-\frac{1}{2}} g_{a}\left(y_{1}\right) .
$$

Suppose $y_{2}$ is the supremum of the values $\tilde{y}_{2}$ until which the solution can be extended and $g_{a}\left(\tilde{y}_{2}\right)>0, g_{a}^{\prime}\left(\tilde{y}_{2}\right)<0$. From Lemma 3.3. we can prove that $\tilde{y}_{2}$ is bounded (We can divide $g_{a}, y \in\left[y_{1}, \tilde{y}_{2}\right]$ into two parts. On the first part $g_{a}$ is bounded, $g_{a}^{\prime}<-\frac{1}{2}$ and on the second part $-\frac{1}{2}<g_{a}^{\prime}<0$ and $k_{a}$ is away from $\frac{1}{2}$ ), hence $y_{2}$ is finite.

From Lemma 3.2 and the monotonicity of $g_{a}$ and $g_{a}^{\prime}$ we know $g_{a}$ can be extended to $y_{2}$ and

$$
\begin{aligned}
& g_{a}\left(y_{2}\right) \geq \mathrm{e}^{-C}\left(1+g_{a}^{\prime}\left(y_{1}\right)^{2}\right)^{-\frac{1}{2}} g_{a}\left(y_{1}\right), \\
& g_{a}^{\prime}\left(y_{2}\right) \leq 0 .
\end{aligned}
$$

So we must have $g_{a}^{\prime}\left(y_{2}\right)=0$, or there would be a contradiction with the fact that $y_{2}$ is the supremum of $\tilde{y}_{2}$. Because $C_{4}<\frac{1}{2}$, we have $0<g_{a}\left(y_{2}\right)<\frac{1}{2}$.

Lemma 3.6. When $a \in[0.9,1.1]$, if (H3) happens, then $\exists y_{1}^{\prime} \in(0,+\infty)$ such that $g_{a}(y)$ can be extended until $\left(0, y_{1}^{\prime}\right)$ but not until $\left(0, y_{1}^{\prime}\right]$. Moreover, in $\left(0, y_{1}^{\prime}\right), g_{a}(y)$ and $g_{a}^{\prime}(y)$ are monotonically decreasing and

$$
\lim _{y \rightarrow y_{1}^{\prime-}} g_{a}(y) \geq 0, \lim _{y \rightarrow y_{1}^{\prime-}} g_{a}^{\prime}(y)=-\infty .
$$

Proof. When $a \in[0.9,1.1]$, we claim, if (H3) happens, when $y>0$, as long as the solution can be extended, $g_{a}(y)>0, g_{a}^{\prime}(y)<0, g_{a}^{\prime \prime}(y)<0$.

First we prove this claim. When $y \in(0, \delta)$ for a small $\delta, g_{a}(y)>0, g_{a}^{\prime}(y)<$ $0, g_{a}^{\prime \prime}(y)<0$ hold. If the claim were false then there would be some $y_{1}>0$ such that

$$
g_{a}\left(y_{1}\right)>0, g_{a}^{\prime}\left(y_{1}\right)<0, g_{a}^{\prime \prime}\left(y_{1}\right)=0
$$

and in $\left(0, y_{1}\right), g_{a}(y)>0, g_{a}^{\prime}(y)<0, g_{a}^{\prime \prime}(y)<0$ hold. From the monotonicity of $g_{a}$ we have

$$
\frac{d \tau}{d g_{a}}=\frac{d \tau}{d y} \frac{1}{g_{a}^{\prime}}=g_{a} \rho
$$

Since $g_{a}$ is bounded, there is $C>0$ such that

$$
\begin{gathered}
\left|\tau\left(g_{a}\left(y_{1}\right), g_{a}^{\prime}\left(y_{1}\right)\right)-\left(a-a^{2}\right)\right|=\left|\tau\left(g_{a}\left(y_{1}\right), g_{a}^{\prime}\left(y_{1}\right)\right)-\tau(a, 0)\right| \leq C H \\
g_{a}\left(y_{1}\right)=\sqrt{\frac{\tau\left(g_{a}\left(y_{1}\right), g_{a}^{\prime}\left(y_{1}\right)\right)}{\frac{1}{k_{a}\left(y_{1}\right)}-1}}=\sqrt{\frac{a-a^{2} \pm C H}{\frac{1}{k_{a}\left(y_{1}\right)}-1}} .
\end{gathered}
$$


Note that $k_{a}\left(y_{1}\right) \in\left[\frac{1}{2+\hat{C} H}, \frac{1}{2-\hat{C} H}\right]$. So we can choose $H$ sufficiently small such that $g_{a}\left(y_{1}\right) \leq \sqrt{\frac{0.9-0.9^{2}+C H}{1-C H}} \approx 0.3$. One can choose $C_{4}$ in Lemma 3.5 such that $\sqrt{\frac{0.9-0.9^{2}+C H}{1-C H}} \leq C_{4} \leq \frac{1}{2(\sqrt{2}+C H)}$. So $g_{a}\left(y_{1}\right) \leq C_{4}$. From Lemma 3.5 one can find a contradiction with (H3). So the claim is true.

Now we prove the lemma. Suppose the solution can be extended to $\left[0, y^{*}\right]$. From $g_{a}\left(y^{*}\right)>0, g_{a}^{\prime}\left(y^{*}\right)<0, g_{a}^{\prime \prime}\left(y^{*}\right)<0$ and Lemma 3.1, the solution can be extended beyond $y^{*}$. So we know the maximal interval in which the solution exists should look like $\left[0, y_{1}^{\prime}\right)$ with $y_{1}^{\prime}$ possibly be $+\infty$. To see that $y_{1}^{\prime}$ is finite, we just prove that when $-\frac{1}{2}<g_{a}^{\prime}<0, k_{a}$ is away from $\frac{1}{2}$. Then we can apply Lemma 3.3. by dividing the orbit into two parts. Note that

$$
k_{a}=g \sqrt{1+g^{\prime 2}}=\frac{1 \pm \sqrt{1-4 \tau\left(1+g^{\prime 2}\right)}}{2} .
$$

So we have

$$
\left|k_{a}-\frac{1}{2}\right|=\frac{\sqrt{1-4 \tau\left(1+g^{\prime 2}\right)}}{2} .
$$

From (19) and $g^{\prime 2} \leq \frac{1}{4}$ we have

$$
\left|k_{a}-\frac{1}{2}\right| \geq \frac{\sqrt{0.55 \pm C H}}{2} .
$$

And from the monotonicity we know $\lim _{y \rightarrow y_{1}^{\prime}} g_{a}(y), \lim _{y \rightarrow y_{1}^{\prime-}} g_{a}^{\prime}(y)$ exist ( $\lim _{y \rightarrow y_{1}^{\prime-}} g_{a}^{\prime}(y)$ is possibly $\left.-\infty\right)$ and

$$
\lim _{y \rightarrow y_{1}^{\prime-}} g_{a}(y) \geq 0
$$

We will see $\lim _{y \rightarrow y_{1}^{\prime-}} g_{a}^{\prime}(y)$ can not be finite. Should it be finite, if $\lim _{y \rightarrow y_{1}^{\prime-}} g_{a}(y)=$ 0 , from (13) one could find $y_{1}<y_{1}^{\prime}$ such that $g_{a}^{\prime \prime}\left(y_{1}\right)=0$ (where $k_{a} \approx \frac{1}{2}$ ) which is a contradiction. If $\lim _{y \rightarrow y_{1}^{\prime-}} g_{a}^{\prime}(y)$ should be finite and $\lim _{y \rightarrow y_{1}^{\prime}} g_{a}(y)>0$, as $y_{1}^{\prime}$ is finite, from Lemma 3.2 and Lemma 3.1 the solution can be extended beyond $y_{1}$ which is also a contradiction. So we have

$$
\lim _{y \rightarrow y_{1}^{-}} g_{a}^{\prime}(y)=-\infty
$$

Lemma 3.7. If (H1) or (H2) happens, there exists $y_{3}<+\infty$ such that $g_{a}$ can be extended to $y_{3}$ and $g_{a}\left(y_{3}\right)>g_{a}\left(y_{2}\right), g_{a}^{\prime}\left(y_{3}\right)>0, g_{a}^{\prime \prime}\left(y_{3}\right)=0$. In $\left[y_{2}, y_{3}\right]$ both $g_{a}$ and $g_{a}^{\prime}$ are monotonically increasing. Moreover, there is $C>0$ which does not depend on a such that

$$
\tau\left(g_{a}\left(y_{3}\right), g_{a}^{\prime}\left(y_{3}\right)\right) \in\left[(1-C H) \tau\left(g_{a}\left(y_{1}\right), g_{a}^{\prime}\left(y_{1}\right)\right),(1+C H) \tau\left(g_{a}\left(y_{1}\right), g_{a}^{\prime}\left(y_{1}\right)\right)\right] .
$$


Proof. So from (18) we know

$$
\frac{d \tau}{d g_{a}^{2}}=\frac{1}{2} \rho
$$

From $|\rho| \leq \hat{C} H$, we have

$$
\left|\tau\left(g_{a}\left(y_{2}\right), g_{a}^{\prime}\left(y_{2}\right)\right)-\tau\left(g_{a}\left(y_{1}\right), g_{a}^{\prime}\left(y_{1}\right)\right)\right| \leq C H g_{a}\left(y_{1}\right)^{2} .
$$

We know

$$
\tau\left(g_{a}\left(y_{1}\right), g_{a}^{\prime}\left(y_{1}\right)\right)=\left(\frac{1}{k_{a}\left(y_{1}\right)}-1\right) g_{a}\left(y_{1}\right)^{2} \in\left[(1-C H) g_{a}\left(y_{1}\right)^{2},(1+C H) g_{a}\left(y_{1}\right)^{2}\right] .
$$

So

$$
\left.\tau\left(g_{a}\left(y_{2}\right), g_{a}^{\prime}\left(y_{2}\right)\right)\right) \in\left[(1-C H) g_{a}\left(y_{1}\right)^{2},(1+C H) g_{a}\left(y_{1}\right)^{2}\right] .
$$

From $g_{a}\left(y_{2}\right) \in\left(0, \frac{1}{2}\right)$ and $g_{a}^{\prime}\left(y_{2}\right)=0$ we can get finer estimate for $g_{a}\left(y_{2}\right)$, i.e.

$$
g_{a}\left(y_{2}\right) \in\left((1-C H) g_{a}\left(y_{1}\right)^{2},(1+C H) g_{a}\left(y_{1}\right)^{2}\right) .
$$

From Lemma 3.1 $g_{a}$ can be extended beyond $y_{2}$. And in a small interval $\left(y_{2}, y_{2}+\delta\right), g_{a}(y)>0, g_{a}^{\prime}(y)>0, g_{a}^{\prime \prime}(y)>0$. We want to show there is $y_{3}>y_{2}$ such that $g_{a}^{\prime \prime}\left(y_{3}\right)=0$ and in $\left(y_{2}, y_{3}\right), g_{a}^{\prime \prime}(y)>0$. If it were false, then as long as $g_{a}$ can be extended and $y>y_{2}$ we would have $g_{a}(y)>0, g_{a}^{\prime}(y)>0, g_{a}^{\prime \prime}(y)>0$. So when $y>y_{2}$, both $g_{a}$ and $g_{a}^{\prime}$ would be monotonically increasing. Suppose $g_{a}$ can be extended to $\left(y_{2}, \tilde{y}_{3}\right)$ but not until $\tilde{y}_{3}$, where $\tilde{y}_{3}$ is possibly $+\infty$. However, as $k_{a}\left(y_{2}\right)=g_{a}\left(y_{2}\right) \leq(1+C H) g_{a}\left(y_{1}\right)^{2} \leq(1+C H) C_{4}^{2} \leq \frac{1}{4},\left\{\left(g_{a}, g_{a}^{\prime}\right) ; y>y_{2}\right\}$ can not intersect with $k_{a}=\frac{2}{3}$ or one would find a point $y_{3}$ such that $g_{a}^{\prime \prime}\left(y_{3}\right)=0$ (where $k_{a} \approx \frac{1}{2}$ ). When $y \rightarrow \tilde{y}_{3}^{-}$, there is a limit for $\left(g_{a}, g_{a}^{\prime}\right)$ which lies in the region bounded by $g_{a}=g_{a}\left(y_{2}\right), g_{a}^{\prime}=0$ and $k_{a}=\frac{2}{3}$. As in the last lemma, it is easy to prove that $\tilde{y}_{3}$ has upper bound and from Lemma 3.2 and Lemma 3.1, we can extend the solution beyond $\tilde{y}_{3}$ which is a contradiction. So there must be a $y_{3}>y_{2}, y_{3}<+\infty$ such that $g_{a}^{\prime \prime}\left(y_{3}\right)=0$. We can choose $y_{3}$ as the minimum of such values, so in $\left(y_{2}, y_{3}\right), g_{a}(y)>0, g_{a}^{\prime}(y)>0, g_{a}^{\prime \prime}(y)>0$.

From (201) we know

$$
\left|\tau\left(g_{a}\left(y_{2}\right), g_{a}^{\prime}\left(y_{2}\right)\right)-\tau\left(g_{a}\left(y_{3}\right), g_{a}^{\prime}\left(y_{3}\right)\right)\right| \leq C H g_{a}^{2}\left(y_{3}\right) .
$$

So we have

$$
g_{a}\left(y_{2}\right) \in\left((1-C H) g_{a}\left(y_{3}\right)^{2},(1+C H) g_{a}\left(y_{3}\right)^{2}\right) .
$$

Together with (22) we can deduce

$$
g_{a}\left(y_{3}\right) \in\left[(1-C H) g_{a}\left(y_{1}\right),(1+C H) g_{a}\left(y_{1}\right)\right]
$$

and

$$
\tau\left(g_{a}\left(y_{3}\right), g_{a}^{\prime}\left(y_{3}\right)\right) \in\left[(1-C H) \tau\left(g_{a}\left(y_{1}\right), g_{a}^{\prime}\left(y_{1}\right)\right),(1+C H) \tau\left(g_{a}\left(y_{1}\right), g_{a}^{\prime}\left(y_{1}\right)\right)\right] .
$$


Lemma 3.8. If (H1) or (H2) happens, $g_{a}$ can be extended to $y_{4}>y_{3}$ and $g_{a}\left(y_{4}\right) \in\left(\frac{1}{2}, 1.2\right), g_{a}^{\prime}\left(y_{4}\right)=0$. In $\left[y_{3}, y_{4}\right], g_{a}$ is monotonically increasing and $g_{a}^{\prime}$ is monotonically decreasing. There is $C>0$ such that

$$
\left|\tau\left(g_{a}\left(y_{4}\right), g_{a}^{\prime}\left(y_{4}\right)\right)-\tau\left(g_{a}\left(y_{3}\right), g_{a}^{\prime}\left(y_{3}\right)\right)\right| \leq C H .
$$

Proof. From Lemma 3.1 $g_{a}$ can be extended beyond $y_{3}$. As long as $g_{a}$ can be extended and $y>y_{3}, g_{a}>0, g_{a}^{\prime}>0$, we are going to prove that $g_{a}^{\prime \prime}<0$. We know in a small interval $\left(y_{3}, y_{3}+\delta\right)$ we have $g_{a}>0, g_{a}^{\prime}>0$. From (15) we know, when $y>y_{3}$, as long as the solution could be extended and $g_{a}>0, g_{a}^{\prime}>0, k_{a}<\frac{2}{2+\hat{C} H}$, $k_{a}$ will monotonically increase. If $k_{a}>\frac{1}{2-\hat{C} H}$, we have

$$
g_{a}^{\prime \prime}=\frac{1}{g}\left(1+g_{a}^{\prime 2}\right)\left(1-(2+\rho) k_{a}\right)<0 .
$$

We are going to prove that when $y>y_{3}, g_{a}>0, g_{a}^{\prime}>0, \frac{1}{2+\rho\left(y_{3}\right)} \leq k_{a} \leq \frac{1}{2-\hat{C} H}$ we still have $g_{a}^{\prime \prime}<0$. From (23) and $\frac{1}{2+\rho\left(y_{3}\right)} \leq k_{a} \leq \frac{1}{2-\hat{C} H}$ we know $g_{a}<C_{4}$ and $g_{a}^{\prime} \geq 1$. Similarly, we can derive (16) and (17) again. So we have $g_{a}^{\prime \prime}(y)<0$. So when $y>y_{3}, g_{a}>0, g_{a}^{\prime}>0$, we have $g_{a}$ is monotonically increasing and $g_{a}^{\prime}$ is monotonically decreasing. So in this interval, $g_{a}$ can be regarded as a decreasing function of $g_{a}^{\prime}$. We claim that, when $y>y_{3}$ and $g_{a}>0, g_{a}^{\prime}>0$, as long as the solution could be extended,

$$
k_{a} \leq 3 \text {. }
$$

To see this, by contradiction, we assume $g_{a}$ can be extended to some $\tilde{y}_{4}>y_{3}$ such that when $y \in\left(y_{3}, \tilde{y}_{4}\right)$ we have $g_{a}(y)>0, g_{a}^{\prime}(y)>0$, and $k_{a}\left(\tilde{y}_{4}\right)>3$. Then there must be $y_{4}^{\prime} \in\left(y_{3}, \tilde{y}_{4}\right)$ such that $k_{a}\left(y_{4}^{\prime}\right)=3$. However if we integral (15) from $y_{4}^{\prime}$ to $\tilde{y}_{4}$ we can get a contradiction. So we proved that $k_{a} \leq 3$. So we have $g_{a} \leq 3$. From the monotonicity of $g_{a}$ and $g_{a}^{\prime}$ and Lemma 3.2, obviously the solution can be extended to some $y_{4}>y_{3}$ such that $g_{a}\left(y_{4}\right) \leq 3, g_{a}^{\prime}\left(y_{4}\right)=0$.

From (20) we know

$$
\left|\tau\left(g_{a}\left(y_{4}\right), g_{a}^{\prime}\left(y_{4}\right)\right)-\tau\left(g_{a}\left(y_{3}\right), g_{a}^{\prime}\left(y_{3}\right)\right)\right| \leq C H .
$$

Lemma 3.9. If (H1) or (H2) happens, there holds

$$
\left|\tau\left(g_{a}\left(y_{4}\right), g_{a}\left(y_{4}\right)\right)-\left(a-a^{2}\right)\right|+\left|g_{a}\left(y_{4}\right)-a\right| \leq C H .
$$

Moreover, if we choose $a \in[0.95,1.05]$ and $H$ very small we have $g_{a}\left(y_{4}\right) \in$ $[0.9,1.1]$.

Proof. From (19) (23) (25) we get the conclusion easily.

Lemma 3.10. If (H1) happens, $g_{a}$ can be extended to $y_{5}>y_{4}$ such that $g_{a}\left(y_{5}\right)>$ $0, g_{a}^{\prime}\left(y_{5}\right)<0, g_{a}^{\prime \prime}\left(y_{5}\right)=0$ and

$$
\left|\tau\left(g_{a}\left(y_{5}\right), g_{a}^{\prime}\left(y_{5}\right)\right)-\tau\left(g_{a}\left(y_{4}\right), g_{a}^{\prime}\left(y_{4}\right)\right)\right| \leq C H .
$$


If (H2) happens, $g_{a}$ can be extended to $y_{5}>y_{4}$ such that

$$
\begin{aligned}
& \lim _{y \rightarrow y_{5}^{-}} g_{a} \geq 0, \\
& \lim _{y \rightarrow y_{5}^{-}} g_{a}^{\prime}=-\infty .
\end{aligned}
$$

In either case, $g_{a}$ and $g_{a}^{\prime}$ are monotonically decreasing in $\left(y_{4}, y_{5}\right)$.

Proof. This lemma can be proved in the same way as Lemma 3.6

\section{Analysis of the singularities}

Define a subset $E(H)$ of $[0,95,1.05]$ which includes all the $a$ values such that (H1) happens to $g_{a}$. And denote $\bar{E}(H)=\{a \in[0.95,1.05]: a \notin E(H)\}$. Obviously, we can choose $H$ sufficiently small such that $0.95 \in E(H), 1.05 \in \bar{E}(H)$. And from the continuous dependence of the solution of ODE on its initial values, $E(H)$ is an open subset of $[0,95,1.05]$. Let

$$
a^{\prime}(H)=\inf \bar{E}(H) .
$$

We have $a^{\prime}(H) \in \bar{E}(H)$ and $a^{\prime}(H)>0.95$ for small $H$.

Lemma 4.1.

$$
\lim _{a \rightarrow a^{\prime}(H)^{-}} \max \left\{\left|g_{a}^{\prime}\left(y_{1}\right)\right|,\left|g_{a}^{\prime}\left(y_{5}\right)\right|\right\}=+\infty .
$$

Proof. If it were not true, we could find a sequence $a_{n}$ which tends to $a^{\prime}(H)^{-}$ such that for any $n$

$$
\max \left\{\left|g_{a_{n}}^{\prime}\left(y_{1}\right)\right|,\left|g_{a_{n}}^{\prime}\left(y_{5}\right)\right|\right\} \leq C(H) .
$$

So $\left(g_{a_{n}}, g_{a_{n}}^{\prime}\right) \in D\left(A_{1}(H), A_{2}(H), A_{3}(H), A_{4}(H)\right)$ from Lemma 3.7, 3.8, 3.9 3.10, So

$$
\left\|g_{a_{n}}(y)\right\|_{C_{y}^{2}\left(\left[0, y_{5}\right]\right)} \leq C(H)
$$

Note that $y_{i}, i=1, \cdots, 5$ depend on $a$ and $H$. Set $y_{0}=0$. We are going to prove that there is $C(H)>0$ such that

$$
C(H)^{-1} \leq y_{i}-y_{i-1} \leq C(H) .
$$

We only prove this for $y_{1}-y_{0}$ and $y_{2}-y_{1}$. The rest inequalities follow similarly. It is easy to see that $k_{a}<3$ and from the same argument as in the proof of Lemma 3.6 we see when $-\frac{1}{2}<g_{a}^{\prime} \leq 0$ on both sides of $y_{1}, k_{a}-\frac{1}{2}$ does not change sign and is bounded away from 0 . By dividing the orbit into $-\frac{1}{2}<g_{a}^{\prime} \leq 0$ and $g_{a}^{\prime} \leq-\frac{1}{2}$, we can apply Lemma 3.3 to get (27).

By passing to a subsequence, we may assume that for fixed $H$, when $a_{n} \rightarrow$ $a^{\prime}(H)^{-}$we have $y_{i} \rightarrow y_{i}^{*}$ for $i=1, \cdots, 5$ and

$$
C(H)^{-1} \leq y_{i}^{*}-y_{i-1}^{*} \leq C(H) .
$$


Differentiate the ODE (6) with respect to $y$ once. From (26) and (16) we know

$$
\left\|g_{a_{n}}\right\|_{C_{y}^{3}\left(\left[0, y_{5}\right]\right)} \leq C(H) \text {. }
$$

So $g_{a_{n}}$ converges to some $\tilde{g}(y), y \in\left[0, y_{5}^{*}-\delta\right]$ in $C^{2}$ for any small $\delta>0$. We know $\tilde{g}(y)$ solves ODE (6) with $a=a^{\prime}(H)$. From the uniqueness of the ODE, we know $g_{a^{\prime}(H)}(y)=\tilde{g}(y), y \in\left[0, y_{5}^{*}\right)$. By uniform $C^{3}$ estimate of $g_{a_{n}}$, we know $g_{a^{\prime}(H)}$ can be extended to $y=y_{5}^{*}$ and $g_{a^{\prime}(H)}^{\prime \prime}\left(y_{5}^{*}\right)=0$ and in each interval $\left[y_{i-1}^{*}, y_{i}^{*}\right]$ it has the same monotonicity as $g_{a}$ in $\left[y_{i-1}, y_{i}\right]$. Then from the proof of Lemma 3.5, we know (H1) happens to $g_{a^{\prime}(H)}$ which is a contradiction. So we proved this lemma.

So there is $\delta_{1}(H)>0$ which depends on $H$ such that when $-\delta_{1}(H)<a-$ $a^{\prime}(H)<0$,

$$
\max \left\{\left|g_{a}^{\prime}\left(y_{1}\right)\right|,\left|g_{a}^{\prime}\left(y_{5}\right)\right|\right\} \geq \frac{1}{\sqrt{H}} .
$$

Lemma 4.2. There is $C>0$ which does not depend on a, $H$, such that when $-\delta_{1}(H)<a-a^{\prime}(H)<0$,

$$
\min \left\{\left|g_{a}^{\prime}\left(y_{1}\right)\right|,\left|g_{a}^{\prime}\left(y_{5}\right)\right|\right\} \geq \frac{C}{\sqrt{H}} .
$$

Proof. Let's first assume

$$
\frac{1}{\sqrt{H}} \leq \max \left\{\left|g_{a}^{\prime}\left(y_{1}\right)\right|,\left|g_{a}^{\prime}\left(y_{5}\right)\right|\right\}=\left|g_{a}^{\prime}\left(y_{1}\right)\right| .
$$

From Lemma 3.7 and $g_{a}\left(y_{1}\right) \sqrt{1+g_{a}^{\prime}\left(y_{1}\right)^{2}} \in\left[\frac{1}{2+\hat{C} H}, \frac{1}{2-\hat{C} H}\right]$ we know there is a uniform $C>0$ such that

$$
\tau\left(g_{a}\left(y_{1}\right), g_{a}^{\prime}\left(y_{1}\right)\right), \tau\left(g_{a}\left(y_{3}\right), g_{a}^{\prime}\left(y_{3}\right)\right) \in\left[C^{-1} H, C H\right] .
$$

From Lemma 3.8 and Lemma 3.10, we know

$$
0<\tau\left(g_{a}\left(y_{5}\right), g_{a}^{\prime}\left(y_{5}\right)\right) \leq C H .
$$

Combining this with $g_{a}\left(y_{5}\right) \sqrt{1+g_{a}^{\prime}\left(y_{5}\right)^{2}} \in\left[\frac{1}{2+\hat{C} H}, \frac{1}{2-\hat{C} H}\right]$, we know there is $C>0$ such that

$$
\left|g_{a}^{\prime}\left(y_{5}\right)\right| \geq \frac{C}{\sqrt{H}}
$$

If

$$
\max \left\{\left|g_{a}^{\prime}\left(y_{1}\right)\right|,\left|g_{a}^{\prime}\left(y_{5}\right)\right|\right\}=\left|g_{a}^{\prime}\left(y_{5}\right)\right|
$$

we can prove the lemma similarly. 
Let $h(y)=\sqrt{1-\left(y-y_{4}\right)^{2}}, y \in\left[y_{4}-1, y_{4}+1\right] . h(y)$ solves the following ODE

$$
\left\{\begin{array}{l}
h^{\prime \prime}(y)-\frac{1}{h(y)}\left(1+h^{\prime}(y)^{2}\right)+2\left(1+h^{\prime}(y)^{2}\right)^{\frac{3}{2}}=0 \\
h\left(y_{4}\right)=1 \\
h^{\prime}\left(y_{4}\right)=0
\end{array}\right.
$$

There is exactly one $y_{6} \in\left(y_{3}, y_{4}\right)$ such that $g_{a}^{\prime}\left(y_{6}\right)=1$. Define two continuous maps

$$
\begin{aligned}
& \Phi_{1}:\left[y_{6}, y_{4}\right] \rightarrow \mathbb{R}, \\
& \Phi_{2}:\left[y_{3}, y_{6}\right] \rightarrow \mathbb{R}
\end{aligned}
$$

such that $g_{a}^{\prime}(y)=h^{\prime}\left(\Phi_{1}(y)\right), \Phi_{1}\left(y_{4}\right)=y_{4}$ and $g_{a}(y)=h\left(\Phi_{2}(y)\right), \Phi_{2}\left(y_{6}\right) \in\left(y_{4}-\right.$ $\left.1, y_{4}\right)$. We use a graph below to illustrate the definition of $\Phi_{1}, \Phi_{2}$. In this graph, the horizontal direction represents $g_{a}^{\prime}(y)$ or $h^{\prime}(y)$ and the vertical direction represents $g_{a}(y)$ or $h(y)$.

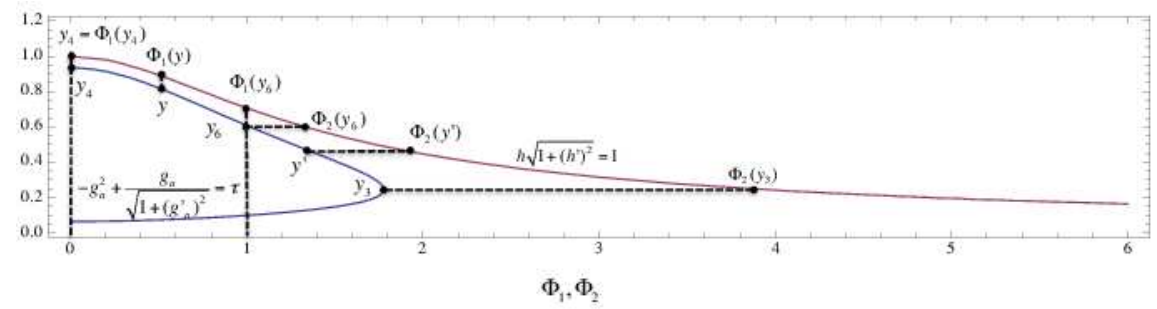

We have the following estimates,

Lemma 4.3. There is $C>0$ such that when $H$ is very small and $-\delta_{1}(H)<$ $a-a^{\prime}(H)<0$,

$$
\begin{aligned}
& \left|\Phi_{1}(y)-y\right| \leq C H, \\
& \left|\Phi_{2}(y)-y\right| \leq C H\left(\frac{H}{g_{a}^{2}(y)}+\left|\log g_{a}(y)\right|\right) .
\end{aligned}
$$

Proof.

$$
\begin{aligned}
\frac{d \Phi_{1}(y)}{d y} & =\frac{d g_{a}^{\prime}(y)}{d y} \frac{d \Phi_{1}(y)}{d h^{\prime}\left(\Phi_{1}(y)\right)} \\
& =\frac{\frac{1}{g_{a}}\left(1+g_{a}^{\prime 2}\right)-\left.(2+\rho)\left(1+g_{a}^{\prime 2}\right)^{\frac{3}{2}}\right|_{y}}{\frac{1}{h}\left(1+h^{\prime 2}\right)-\left.2\left(1+h^{\prime 2}\right)^{\frac{3}{2}}\right|_{\Phi_{1}(y)}} .
\end{aligned}
$$

When $-\delta_{1}(H)<a-a^{\prime}(H)<0,\left|\tau\left(g_{a}(y), g_{a}^{\prime}(y)\right)\right| \leq C H, y \in\left[0, y_{5}\right]$. So we know when $y \in\left[y_{6}, y_{4}\right], g_{a}(y) \in\left[h\left(\Phi_{1}(y)\right)(1-C H), h\left(\Phi_{1}(y)\right)(1+C H)\right]$. Hence,

$$
\left|\frac{\frac{1}{g_{a}}\left(1+g_{a}^{\prime 2}\right)-\left.(2+\rho)\left(1+g_{a}^{\prime 2}\right)^{\frac{3}{2}}\right|_{y}}{\frac{1}{h}\left(1+h^{\prime 2}\right)-\left.2\left(1+h^{\prime 2}\right)^{\frac{3}{2}}\right|_{\Phi_{1}(y)}}-1\right| \leq C H .
$$


So the first estimate follows. For the second inequality, first we notice

$$
\left|h\left(\Phi_{1}\left(y_{6}\right)\right)-h\left(\Phi_{2}\left(y_{6}\right)\right)\right|=\left|h\left(\Phi_{1}\left(y_{6}\right)\right)-g_{a}\left(y_{6}\right)\right| \leq C H .
$$

From $h^{\prime}\left(\Phi_{1}\left(y_{6}\right)\right)=1$, we know $\Phi_{1}\left(y_{6}\right)=y_{4}-\frac{\sqrt{2}}{2}$. So $h\left(\Phi_{1}\left(y_{6}\right)\right)=\frac{\sqrt{2}}{2}$ and

$$
\left|\Phi_{2}\left(y_{6}\right)-\Phi_{1}\left(y_{6}\right)\right| \leq C H \text {. }
$$

When $y \in\left[y_{3}, y_{6}\right]$,

$$
\begin{aligned}
y-y_{6} & =\int_{g_{a}\left(y_{6}\right)}^{g_{a}(y)} \frac{1}{g_{a}^{\prime}} d g_{a} \\
& =\int_{g_{a}\left(y_{6}\right)}^{g_{a}(y)} \frac{\tau+g_{a}^{2}}{\sqrt{g_{a}^{2}-\left(\tau+g_{a}^{2}\right)^{2}}} d g_{a}
\end{aligned}
$$

and

$$
\begin{aligned}
\Phi_{2}(y)-\Phi_{2}\left(y_{6}\right) & =\int_{h\left(\Phi_{2}\left(y_{6}\right)\right)}^{h\left(\Phi_{2}(y)\right)} \frac{1}{h^{\prime}} d h \\
& =\int_{g_{a}\left(y_{6}\right)}^{g_{a}(y)} \frac{g_{a}}{\sqrt{1-g_{a}^{2}}} d g_{a} .
\end{aligned}
$$

So we have

$$
\begin{aligned}
& \Phi_{2}(y)-y-\left(\Phi_{2}\left(y_{6}\right)-y_{6}\right) \\
= & \int_{g_{a}\left(y_{6}\right)}^{g_{a}(y)}\left(\frac{g_{a}}{\sqrt{1-g_{a}^{2}}}-\frac{\tau+g_{a}^{2}}{\sqrt{g_{a}^{2}-\left(\tau+g_{a}^{2}\right)^{2}}}\right) d g_{a} \\
= & \int_{g_{a}\left(y_{6}\right)}^{g_{a}(y)} \frac{-\tau^{2}-2 \tau g_{a}^{2}}{\sqrt{1-g_{a}^{2}} \sqrt{g_{a}^{2}-\left(\tau+g_{a}^{2}\right)^{2}}\left(g_{a} \sqrt{g_{a}^{2}-\left(\tau+g_{a}^{2}\right)^{2}}+\left(\tau+g_{a}^{2}\right) \sqrt{1-g_{a}^{2}}\right)} d g_{a} .
\end{aligned}
$$

When $y \in\left[y_{3}, y_{6}\right]$,

$$
\begin{aligned}
1-g_{a}^{2} & \geq 1-\left(\frac{\sqrt{2}}{2}+C H\right)^{2} \geq \frac{1}{3}, \\
|\tau| & \leq C H .
\end{aligned}
$$

From (24) we know when $y \in\left[y_{3}, y_{4}\right]$

$$
\frac{\tau+g_{a}^{2}}{g_{a}^{2}}=\frac{1}{k_{a}} \geq \frac{1}{3}
$$

So

$$
\tau+g_{a}^{2} \geq \frac{g_{a}^{2}}{3} .
$$

From $g_{a} \leq \frac{\sqrt{2}}{2}+C H,|\tau| \leq C H$, one can check that, for some $C>0$,

$$
g_{a}^{2}-\left(\tau+g_{a}^{2}\right)^{2} \geq C g_{a}^{2} .
$$


So we have

$$
\left|\frac{-\tau^{2}-2 \tau g_{a}^{2}}{\sqrt{1-g_{a}^{2}} \sqrt{g_{a}^{2}-\left(\tau+g_{a}^{2}\right)^{2}}\left(g_{a} \sqrt{g_{a}^{2}-\left(\tau+g_{a}^{2}\right)^{2}}+\left(\tau+g_{a}^{2}\right) \sqrt{1-g_{a}^{2}}\right)}\right| \leq C \frac{H^{2}+H g_{a}^{2}}{g_{a}^{3}} .
$$

Then

$$
\begin{aligned}
\left|\Phi_{2}(y)-y-\left(\Phi_{2}\left(y_{6}\right)-y_{6}\right)\right| & \leq C\left|\int_{g_{a}\left(y_{6}\right)}^{g_{a}(y)} \frac{H^{2}+H g_{a}^{2}}{g_{a}^{3}} d g_{a}\right| \\
& =C\left(\left|\frac{H^{2}}{g_{a}^{2}(y)}-\frac{H^{2}}{g_{a}^{2}\left(y_{6}\right)}\right|+H\left|\log g_{a}(y)-\log g_{a}\left(y_{6}\right)\right|\right) \\
& \leq C H\left(\frac{H}{g_{a}^{2}(y)}+\left|\log g_{a}(y)\right|\right)
\end{aligned}
$$

So we have when $y \in\left[y_{3}, y_{6}\right]$

$$
\begin{aligned}
\left|\Phi_{2}(y)-y\right| & \leq\left|\Phi_{2}\left(y_{6}\right)-\Phi_{1}\left(y_{6}\right)\right|+\left|\Phi_{1}\left(y_{6}\right)-y_{6}\right|+C H\left(\frac{H}{g_{a}^{2}}+\left|\log g_{a}\right|\right) \\
& \leq C H\left(\frac{H}{g_{a}^{2}}+\left|\log g_{a}\right|+1\right) .
\end{aligned}
$$

As $1 \leq C\left|\log \frac{\sqrt{2}}{2}\right|$, we get the second estimate.

Lemma 4.4. When $H$ is very small and $-\delta_{1}(H)<a-a^{\prime}(H)<0$,

$$
\begin{aligned}
& \left|y_{4}-2\right| \leq C \sqrt{H} \\
& \left|y_{5}-3\right| \leq C \sqrt{H} .
\end{aligned}
$$

Proof. From (23) we know

$$
h\left(\Phi_{2}\left(y_{3}\right)\right)=g_{a}\left(y_{3}\right) \leq C \sqrt{H} .
$$

So there is some $y_{6}^{\prime} \in\left(y_{3}, y_{6}\right)$ such that $g_{a}\left(y_{6}^{\prime}\right)=C \sqrt{H}$ where we use the same constant $C$ as the right hand side of the above inequality. From Lemma 4.3, we know

$$
\left|y_{6}^{\prime}-\Phi_{2}\left(y_{6}^{\prime}\right)\right| \leq C H\left(\frac{H}{g_{a}^{2}\left(y_{6}^{\prime}\right)}+\log g_{a}\left(y_{6}^{\prime}\right)\right) \leq C H|\log H| .
$$

We know that $\left|\Phi_{2}\left(y_{6}^{\prime}\right)-\left(y_{4}-1\right)\right| \leq C H$. So we have

$$
\left|y_{6}^{\prime}-\left(y_{4}-1\right)\right| \leq C H|\log H| .
$$

By applying Lemma 3.3 we can get

$$
\left|y_{6}^{\prime}-y_{2}\right| \leq C \sqrt{H}
$$


In the same way we can find $\hat{y}_{1} \in\left(1, y_{1}\right)$ such that $g_{a}\left(\hat{y}_{1}\right)=C \sqrt{H}$ for some $C>0$. And we can prove that

$$
\begin{aligned}
\left|\hat{y}_{1}-1\right| & \leq C H \log H, \\
\left|\hat{y}_{1}-y_{2}\right| & \leq C \sqrt{H} .
\end{aligned}
$$

So

$$
\begin{aligned}
\left|y_{4}-2\right| & =\left|y_{4}-1-y_{6}^{\prime}+y_{6}^{\prime}-y_{2}+y_{2}-\hat{y}_{1}+\hat{y}_{1}-1\right| \\
& \leq C H \log H+C \sqrt{H} \leq C \sqrt{H} .
\end{aligned}
$$

The second estimate follows similarly.

Lemma 4.5. When $H$ is very small and $-\delta_{1}(H)<a-a^{\prime}(H)<0$,

$$
\begin{array}{r}
\left|\int_{y_{3}}^{y_{4}} \frac{1}{\sqrt{y^{2}+g_{a}(y)^{2}}} g_{a} g_{a}^{\prime} d y-\int_{y_{4}-1}^{y_{4}} \frac{1}{\sqrt{y^{2}+h(y)^{2}}} h h^{\prime} d y\right| \leq C H, \\
\left|\int_{y_{3}}^{y_{4}} \frac{g_{a}-y g_{a}^{\prime}}{\left(y^{2}+g_{a}(y)^{2}\right)^{\frac{3}{2}} \sqrt{1+g_{a}^{\prime 2}}} g_{a} g_{a}^{\prime} d y-\int_{y_{4}-1}^{y_{4}} \frac{h-y h^{\prime}}{\left(y^{2}+h(y)^{2}\right)^{\frac{3}{2}} \sqrt{1+h^{\prime 2}}} h h^{\prime} d y\right| \leq C H .
\end{array}
$$

Proof. For the first one we have an obvious reason to prove

$$
\left|\int_{y_{6}^{\prime}}^{y_{4}} \frac{1}{\sqrt{y^{2}+g_{a}(y)^{2}}} g_{a} g_{a}^{\prime} d y-\int_{\Phi_{2}\left(y_{6}^{\prime}\right)}^{y_{4}} \frac{1}{\sqrt{y^{2}+h(y)^{2}}} h h^{\prime} d y\right| \leq C H
$$

instead, where $y_{6}^{\prime} \in\left(y_{3}, y_{6}\right)$ and $g_{a}\left(y_{6}^{\prime}\right)=C \sqrt{H}$. By using Lemma 4.3, this can be verified as

$$
\begin{aligned}
&\left|\int_{y_{6}^{\prime}}^{y_{6}} \frac{1}{\sqrt{y^{2}+g_{a}(y)^{2}}} g_{a} g_{a}^{\prime} d y-\int_{\Phi_{2}\left(y_{6}^{\prime}\right)}^{\Phi_{2}\left(y_{6}\right)} \frac{1}{\sqrt{y^{2}+h(y)^{2}}} h h^{\prime} d y\right| \\
& \leq \int_{g_{a}\left(y_{6}^{\prime}\right)}^{g_{a}\left(y_{6}\right)}\left|\frac{g_{a}}{\sqrt{y^{2}+g_{a}(y)^{2}}}-\frac{g_{a}}{\sqrt{\Phi_{2}(y)^{2}+g_{a}(y)^{2}}}\right| d g_{a} \\
& \leq C \int_{g_{a}\left(y_{6}^{\prime}\right)}^{g_{a}\left(y_{6}\right)} g_{a} H\left(\frac{H}{g_{a}^{2}}+\left|\log g_{a}\right|\right) d g_{a} \\
& \leq C H^{2}|\log H|+C H \leq C H, \\
& \mid \int_{\Phi_{1}\left(y_{6}\right)}^{y_{4}} \quad \quad \int_{\Phi_{2}\left(y_{6}\right)}^{\Phi_{1}\left(y_{6}\right)} \frac{1}{\sqrt{y^{2}+h(y)^{2}}} h h^{\prime} d y-\int_{y_{6}}^{y_{4}} \frac{1}{\sqrt{y^{2}+g_{a}(y)^{2}}} g_{a} g_{a}^{\prime} d y \mid \\
& \leq\left|\int_{y_{6}}^{y_{4}} \frac{1}{\sqrt{\Phi_{1}(y)^{2}+h\left(\Phi_{1}(y)\right)^{2}}} h\left(\Phi_{1}(y)\right) h^{\prime}\left(\Phi_{1}(y)\right) \Phi_{1}^{\prime}(y)-\frac{1}{\sqrt{y^{2}+g_{a}(y)^{2}}} g_{a} g_{a}^{\prime} d y\right| \\
& \leq C H .
\end{aligned}
$$


The proof of the second one is similar.

From Lemma 4.2. using the same technique as the above lemma, we can prove

Lemma 4.6. When $H$ is very small and $-\delta_{4}(H)<a-a^{\prime}(H)<0$, we have

$$
\begin{array}{r}
\left|\int_{y_{4}}^{y_{5}} \frac{1}{\sqrt{y^{2}+g_{a}(y)^{2}}} g_{a} g_{a}^{\prime} d y-\int_{y_{4}}^{y_{4}+1} \frac{1}{\sqrt{y^{2}+h(y)^{2}}} h h^{\prime} d y\right| \leq C H, \\
\left|\int_{y_{4}}^{y_{5}} \frac{g_{a}-y g_{a}^{\prime}}{\left(y^{2}+g_{a}(y)^{2}\right)^{\frac{3}{2}} \sqrt{1+g_{a}^{\prime 2}}} g_{a} g_{a}^{\prime} d y-\int_{y_{4}}^{y_{4}+1} \frac{h-y h^{\prime}}{\left(y^{2}+h(y)^{2}\right)^{\frac{3}{2}} \sqrt{1+h^{\prime 2}}} h h^{\prime} d y\right| \leq C H .
\end{array}
$$

Lemma 4.7. We can fix $\lambda>0$ small and $p>0$ large such that there is $\delta(p, \lambda)>0$ small such that when $0<H<\delta(p, \lambda)$ and $-\delta_{1}(H)<a-a^{\prime}(H)<0$, we have $\left|g_{a}^{\prime}\left(y_{5}\right)\right| \geq\left|g_{a}^{\prime}\left(y_{1}\right)\right|$. And moreover

$$
\lim _{a \rightarrow a^{\prime}(H)^{-}} g_{a}^{\prime}\left(y_{5}\right)=-\infty, \liminf _{a \rightarrow a^{\prime}(H)^{-}} g_{a}^{\prime}\left(y_{1}\right)>-\infty .
$$

Proof. From Lemma 4.5 and Lemma 4.6 we know

$$
\begin{aligned}
& \quad \mid \int_{y_{3}}^{y_{5}}\left(\frac{1}{\sqrt{y^{2}+g_{a}(y)^{2}}}+\frac{\left(g_{a}-y g_{a}^{\prime}\right)}{\left(y^{2}+g_{a}(y)^{2}\right)^{\frac{3}{2}} \sqrt{1+g_{a}^{\prime 2}}}\right) g_{a} g_{a}^{\prime} d y \\
& \quad-\int_{y_{4}-1}^{y_{4}+1}\left(\frac{1}{\sqrt{y^{2}+h(y)^{2}}}+\frac{h-y h^{\prime}}{\left(y^{2}+h(y)^{2}\right)^{\frac{3}{2}} \sqrt{1+h^{\prime 2}}}\right) h h^{\prime} d y \mid \\
& \leq C H .
\end{aligned}
$$

The following facts are the key to our proof. When $y_{4}>1$,

$$
\begin{aligned}
\int_{y_{4}-1}^{y_{4}+1}\left(\frac{1}{\sqrt{y^{2}+h(y)^{2}}}+\frac{h-y h^{\prime}}{\left(y^{2}+h(y)^{2}\right)^{\frac{3}{2}} \sqrt{1+h^{\prime 2}}}\right) h h^{\prime} d y=0, \\
\int_{y_{4}-1}^{y_{4}+1} \frac{1}{\sqrt{y^{2}+h(y)^{2}}} h h^{\prime} d y=\frac{2}{3 y_{4}^{2}}, \\
\int_{y_{4}-1}^{y_{4}+1} \frac{h-y h^{\prime}}{\left(y^{2}+h(y)^{2}\right)^{\frac{3}{2}} \sqrt{1+h^{2}}} h h^{\prime} d y=-\frac{2}{3 y_{4}^{2}} .
\end{aligned}
$$

The proof of these facts takes direct calculations which we omit here.

From Lemma 4.4, we know $\left|y_{4}-2\right| \leq C \sqrt{H}$. So

$$
\int_{y_{4}-1}^{y_{4}+1} \frac{h-y h^{\prime}}{\left(y^{2}+h(y)^{2}\right)^{\frac{3}{2}} \sqrt{1+h^{\prime 2}}} h h^{\prime} d y=-\frac{1}{6} \pm O(\sqrt{H}) .
$$

So we have

$$
\left|\int_{y_{3}}^{y_{5}}\left(\frac{H}{\sqrt{y^{2}+g_{a}(y)^{2}}}+\frac{H\left(g_{a}-y g_{a}^{\prime}\right)}{\left(y^{2}+g_{a}(y)^{2}\right)^{\frac{3}{2}} \sqrt{1+g_{a}^{\prime 2}}}\right) g_{a} g_{a}^{\prime} d y\right| \leq C H^{2} .
$$


and when $p>3$

$$
\begin{aligned}
& \int_{y_{3}}^{y_{5}} \frac{(p-3) H^{2}\left(g_{a}-y g_{a}^{\prime}\right)}{\left(y^{2}+g_{a}(y)^{2}\right)^{\frac{3}{2}} \sqrt{1+g_{a}^{\prime 2}}} g_{a} g_{a}^{\prime} d y \leq-(p-3) H^{2}\left(\frac{1}{6}+C H\right) \\
& \leq-\frac{(p-3)}{7} H^{2} \\
&\left|\int_{y_{3}}^{y_{5}} \frac{(\phi-p) H^{2}\left(g_{a}-y g_{a}^{\prime}\right)}{\left(y^{2}+g_{a}(y)^{2}\right)^{\frac{3}{2}} \sqrt{1+g_{a}^{\prime 2}}} g_{a} g_{a}^{\prime} d y\right| \leq C|p-1| H^{2} \lambda^{2}
\end{aligned}
$$

From (8)

$$
\left|\int_{y_{3}}^{y_{5}}\left(-\frac{H^{2} \phi^{\prime}\left(y+g_{a} g_{a}^{\prime}\right)}{8 d^{2} y^{2} \sqrt{1+g_{a}^{\prime 2}}}+C(p, \lambda) O\left(H^{3}\right)\right) g_{a} g_{a}^{\prime} d y\right| \leq C|p-1| \lambda H^{2}+C(p, \lambda) \lambda^{2} H^{3} .
$$

Also note that

$$
\begin{aligned}
\left|\int_{y_{1}}^{y_{3}} g_{a} g_{a}^{\prime} \rho d y\right| & =\left|\int_{g_{a}\left(y_{1}\right)}^{g_{a}\left(y_{2}\right)}+\int_{g_{a}\left(y_{2}\right)}^{g_{a}\left(y_{3}\right)} \frac{1}{2} \rho d g_{a}^{2}\right| \\
& \leq C H^{2} .
\end{aligned}
$$

So we can fix $\lambda>0$ small and $p>0$ large, and there is $\delta(\lambda, p)>0$ small that when $0<H<\delta(\lambda, p)$

$$
\begin{aligned}
& -C p H^{2} \leq \tau\left(g_{a}\left(y_{5}\right), g_{a}^{\prime}\left(y_{5}\right)\right)-\tau\left(g_{a}\left(y_{1}\right), g_{a}^{\prime}\left(y_{1}\right)\right)=\int_{y_{1}}^{y_{5}} \rho g_{a} g_{a}^{\prime} d y \leq-H^{2}, \\
& -C p H^{2} \leq \tau\left(g_{a}\left(y_{5}\right), g_{a}^{\prime}\left(y_{5}\right)\right)-\tau\left(g_{a}\left(y_{3}\right), g_{a}^{\prime}\left(y_{3}\right)\right)=\int_{y_{3}}^{y_{5}} \rho g_{a} g_{a}^{\prime} d y \leq-H^{2} .
\end{aligned}
$$

From $\tau\left(g_{a}\left(y_{i}\right), g_{a}^{\prime}\left(y_{i}\right)\right)>0, i=1,3,5$, we know

$$
\tau\left(g_{a}\left(y_{i}\right), g_{a}^{\prime}\left(y_{i}\right)\right) \geq H^{2}, i=1,3 .
$$

Note that $k_{a}\left(y_{i}\right)=\frac{1}{2 \pm O(H)}, i=1,3,5$. So

$$
\begin{aligned}
& g_{a}^{\prime}\left(y_{i}\right)=\frac{-\sqrt{\tau-4 \tau^{2}}}{2 \tau}=-\frac{1}{2 \sqrt{\tau}}(1+O(\tau)), i=1,5, \\
& g_{a}^{\prime}\left(y_{3}\right)=\frac{1}{2 \sqrt{\tau}}(1+O(\tau)) .
\end{aligned}
$$

So we have

$$
\begin{aligned}
& 0>g_{a}^{\prime}\left(y_{1}\right) \geq-\frac{C}{H}, \\
& 0<g_{a}^{\prime}\left(y_{3}\right) \leq \frac{C}{H},
\end{aligned}
$$

and

$$
\lim _{a \rightarrow a^{\prime}(H)^{-}} g_{a}^{\prime}\left(y_{5}\right)=-\infty
$$


Now we can analyze the singular limit of $g_{a}$ as $a \rightarrow a^{\prime}(H)^{-}$.

Lemma 4.8. Choose $p, \lambda, \delta(p, \lambda), \delta_{1}(H)$ such that the conclusion of Lemma 4.7 holds. When $a \rightarrow a^{\prime}(H)^{-}$, by passing to a sequence, we have $y_{i} \rightarrow y_{i}^{*}, i=1, \cdots, 5$ and $g_{a}$ converges to $g_{a^{\prime}(H)}$ in $C^{2}$ sense on every $[0, \tilde{y}] \subset\left[0, y_{5}^{*}\right)$. (H2) happens to $g_{a^{\prime}(H)}(y)$ and

$$
\begin{aligned}
& \lim _{y \rightarrow y_{5}^{*-}} g_{a^{\prime}(H)}(y)=0, \\
& \lim _{y \rightarrow y_{5}^{*-}} g_{a^{\prime}(H)}^{\prime}(y)=-\infty .
\end{aligned}
$$

Proof. From

$$
\lim _{a \rightarrow a^{\prime}(H)^{-}} g_{a}^{\prime}\left(y_{5}\right)=-\infty
$$

and $g_{a}^{\prime}$ is monotonically decreasing on $\left[y_{4}, y_{5}\right]$ we know for any $j \in \mathbb{N}^{+}$when $a$ is sufficiently close to $a^{\prime}(H)$, there is exactly one $y_{5}^{j} \in\left(y_{4}, y_{5}\right)$ such that $g_{a}^{\prime}\left(y_{5}^{j}\right)=-j$. From

$$
-\frac{C}{H} \leq g_{a}^{\prime}\left(y_{1}\right)<0<g_{a}^{\prime}\left(y_{3}\right) \leq \frac{C}{H}
$$

we know that $g_{a}^{\prime}$ is bounded independent of $a$ on $\left[0, y_{5}^{j}\right]$. From the analysis of the last section, it is not hard to prove that $g_{a}$ has both positive upper bound and positive lower bound on $\left[0, y_{5}^{j}\right]$. So $\left(g_{a}, g_{a}^{\prime}\right) \in D\left(A_{1}, A_{2}, A_{3}, A_{4}\right)$, where $A_{i}=$ $A_{i}(H, j), i=1, \cdots, 4$. So on $\left[0, y_{5}^{j}\right], g_{a}$ has uniform $C^{3}$ bounds. By using the same argument as in Lemma 4.1, we can prove that there is $C(p, \lambda, H)>0$ such that

$$
C(p, \lambda, H)^{-1} \leq y_{i}-y_{i-1} \leq C(p, \lambda, H), i=1, \cdots, 4 .
$$

And from Lemma 3.3, we can prove that $C^{-1}<y_{5}^{j}-y_{4}<C$. So by passing to a sequence, we can assume $y_{i} \rightarrow y_{i}^{*}, i=1, \cdots, 4, y_{5}^{j} \rightarrow y_{5}^{j *}$. And $g_{a}$ converges to $g_{a^{\prime}(H)}$ in $C^{2}\left(\left[0, y_{5}^{j *}\right]\right)$ sense, where $g_{a^{\prime}(H)}$ solves (6) with initial value $a^{\prime}(H)$. By a diagonal method, we can choose $a_{i} \rightarrow a^{\prime}(H)^{-}$such that for any $j \in \mathbb{N}^{+}$

$$
\lim _{i \rightarrow+\infty} y_{5}^{j}=y_{5}^{j *} \text {. }
$$

It is obvious that $y_{5}^{j *}$ is monotonically increasing in $j$. Note that we have

$$
y_{5}^{j+1}-y_{5}^{j}=\int_{-(j+1)}^{-j} \frac{g_{a} d g_{a}^{\prime}}{\left(1+g_{a}^{\prime 2}\right)\left((2+\rho) k_{a}-1\right)} .
$$

For any $j>0$, when $-j-1<g_{a}^{\prime}<-j$, we can choose $a$ close to $a^{\prime}(H)$ such that $k_{a}$ is bounded away from (and bigger than) $\frac{1}{2}$. So there is $C_{j}>0$ such that

$$
y_{5}^{j+1}-y_{5}^{j} \geq C_{j} .
$$

So we know $\left|y_{5}^{j+1 *}-y_{5}^{j *}\right| \geq C_{j}$. From the fact that the second derivative of $g_{a}$ has uniform bound in $\left[0, y_{5}^{j}\right]$, we know

$$
g_{a^{\prime}(H)}^{\prime}\left(y_{5}^{j *}\right)=\lim _{a \rightarrow a^{\prime}(H)^{-}} g_{a}^{\prime}\left(y_{5}^{j *}\right)=\lim _{a \rightarrow a^{\prime}(H)^{-}} g_{a}^{\prime}\left(y_{5}^{j}\right)=-j .
$$


So we know

$$
\lim _{j \rightarrow+\infty} g_{a^{\prime}(H)}^{\prime}\left(y_{5}^{j *}\right)=-\infty
$$

Note that

$$
\left|y_{5}^{j}-y_{5}\right| \leq \frac{C}{j}
$$

If we take a subsequence further, we have $y_{5} \rightarrow y_{5}^{*}$. So we have $\left|y_{5}^{j *}-y_{5}^{*}\right| \leq \frac{C}{j}$. So we have

$$
\lim _{j \rightarrow+\infty} y_{5}^{j *}=y_{5}^{*} .
$$

From the monotonicity of $g_{a}^{\prime}$ we know

$$
\lim _{y \rightarrow y_{5}^{*}} g_{a^{\prime}(H)}^{\prime}(y)=-\infty .
$$

For $k_{a}=g_{a} \sqrt{1+g_{a}^{\prime 2}}$, by analyzing (15), we know when $y \in\left[y_{4}, y_{5}\right]$,

$$
\frac{1}{2+C H} \leq k_{a} \leq \frac{1}{1-C H}
$$

So we have

$$
0<g_{a}\left(y_{5}^{j}\right) \leq \frac{C}{j}
$$

hence $g_{a^{\prime}(H)}\left(y_{5}^{j *}\right) \leq \frac{C}{j}$ and

$$
\lim _{y \rightarrow y_{5}^{*}} g_{a^{\prime}(H)}(y)=0 .
$$

At last, noticing that any $[0, \tilde{y}] \subset\left[0, y_{5}^{*}\right)$ is contained in some $\left[0, y_{5}^{j}\right)$ for any $a$ close to $a^{\prime}(H)$. So we can prove the uniform convergence on $[0, \tilde{y}]$. Obviously, (H2) happens to $g_{a^{\prime}(H)}$.

Here we draw a graph of $g_{a^{\prime}(H)}(y), y \in\left[0, y_{5}^{*}\right)$.

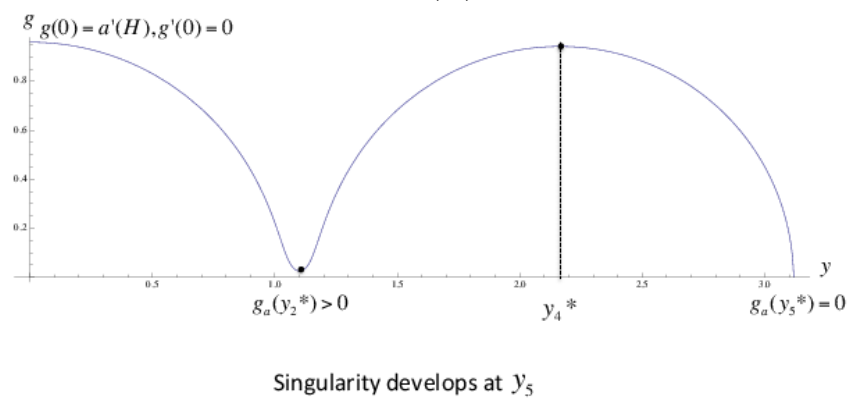

$g_{a^{\prime}(H)}(y)$ is monotonically decreasing in $\left[y_{4}^{*}, y_{5}^{*}\right)$. Now we use $g$ to represent $g_{a^{\prime}(H)}$ for short. Suppose $y=y(g), g \in\left(0, g_{a^{\prime}(H)}\left(y_{4}^{*}\right)\right]$ is the inverse function of 
$g_{a^{\prime}(H)}(y), y \in\left[y_{4}^{*}, y_{5}^{*}\right)$. We make even extension to $y(g)$.

$$
y^{\text {even }}(g)= \begin{cases}y(g), & g \in\left(0, g_{a^{\prime}(H)}\left(y_{4}^{*}\right)\right], \\ y_{5}^{*}, & g=0, \\ y(-g), & g \in\left[-g_{a^{\prime}(H)}\left(y_{4}^{*}\right), 0\right) .\end{cases}
$$

Then we have

Lemma 4.9. $y^{\text {even }}(g)$ has continuous second derivative in $(-\delta, \delta)$ for small $\delta$.

Proof. $y^{\text {even }}(g)$ is continuous at 0 . When $g>0$,

$$
\frac{d y^{\text {even }}}{d g}=\frac{1}{g^{\prime}} \text {. }
$$

From (31), we know when $g$ is close to $0, g^{\prime} \sim \frac{1}{g}$. So we have

$$
\lim _{g \rightarrow 0^{+}} \frac{d y^{\text {even }}}{d g}=0 \text {. }
$$

So $y^{\text {even }}(g)$ has 0 derivative at $g=0$. For the second derivative, we note that

$$
\begin{aligned}
\frac{d^{2} y^{\text {even }}}{d g^{2}} & =\frac{d}{d g}\left(\frac{1}{g^{\prime}}\right)=\frac{-g^{\prime \prime}}{g^{\prime 3}} \\
& =-\frac{1+g^{\prime 2}}{g^{\prime 2}} \frac{1}{g} \frac{1}{g^{\prime}}+(2+\rho) \frac{\left(1+g^{\prime 2}\right)^{\frac{3}{2}}}{g^{\prime 3}} .
\end{aligned}
$$

Regard $\frac{1+g^{\prime 2}}{g^{\prime 2}}$ as $G_{1}(g)$ and $\frac{\left(1+g^{\prime 2}\right)^{\frac{3}{2}}}{g^{\prime 3}}$ as $G_{2}(g)$. We get a linear ODE of first order,

$$
\left\{\begin{array}{l}
\frac{d}{d g}\left(\frac{1}{g^{\prime}}\right)+\frac{G_{1}(g)}{g} \frac{1}{g^{\prime}}=(2+\rho) G_{2}(g), \\
\frac{1}{g^{\prime}}(0)=0,
\end{array}\right.
$$

with

$$
\begin{aligned}
\lim _{g \rightarrow 0^{+}} G_{1}(g) & =1, \\
G_{1}(g) & =1+O\left(g^{2}\right), \\
\lim _{g \rightarrow 0^{+}} G_{2}(g) & =1 .
\end{aligned}
$$

The solution is

$$
\frac{1}{g^{\prime}}=\frac{\int_{0}^{g}(2+\rho) G_{2}(s) s \exp \left(O\left(s^{2}\right)\right) d s}{g \exp \left(O\left(g^{2}\right)\right)} .
$$

So

$$
\begin{aligned}
\lim _{g \rightarrow 0^{+}} \frac{1}{g g^{\prime}} & =\lim _{g \rightarrow 0^{+}} \frac{\int_{0}^{g}(2+\rho) G_{2}(s) s \exp \left(O\left(s^{2}\right)\right) d s}{g^{2} \exp \left(O\left(g^{2}\right)\right)} \\
& =\lim _{g \rightarrow 0^{+}} \frac{(2+\rho) G_{2}(g) g \exp \left(O\left(g^{2}\right)\right)}{2 g \exp \left(O\left(g^{2}\right)\right)+g^{2} \exp \left(O\left(g^{2}\right)\right) O(g)} \\
& =1+\frac{1}{2} \lim _{g \rightarrow 0^{+}} \rho .
\end{aligned}
$$


From (7) we know as $y \rightarrow y_{5}^{*}, g_{a^{\prime}(H)} \rightarrow 0^{+}, g_{a^{\prime}(H)}^{\prime} \rightarrow-\infty$, we have $d \rightarrow y_{5}^{*}, \frac{g_{a}-y g_{a}^{\prime}}{\sqrt{1+g_{a}^{\prime 2}}} \rightarrow$ $y_{5}^{*}$. So the limit

$$
\lim _{g \rightarrow 0^{+}} \rho
$$

exists. So from (32), $\frac{d^{2} y^{\text {even }}}{d g^{2}}(0)$ exists.

\section{Proof of the main theorems}

Now we prove Theorem 1.1. For any $H$ sufficiently small, we have got $g_{a^{\prime}(H)}(y)$, where we have assume that $g_{a^{\prime}(H)}\left(y_{5}^{*}\right)=0$. Consider the surfaces of revolution $\Sigma^{+}(H)$ defined by

$$
r=\frac{2}{H} g_{a^{\prime}(H)}\left(\frac{H}{2} x\right), x \in\left[0, \frac{2 y_{5}^{*}}{H}\right] .
$$

From our construction, when $x \in\left[0, \frac{2 y_{5}^{*}}{H}\right)$ the surface has constant mean curvature $H$. When $x=\frac{2 y_{5}^{*}}{H}$, from Lemma 4.9, the surface actually has well defined and continuous mean curvature at this single point. So the mean curvature at this point must be $H$. Let $\Sigma^{-}(H)$ be defined by

$$
r=\frac{2}{H} g_{a^{\prime}(H)}\left(-\frac{H}{2} x\right), x \in\left[-\frac{2 y_{5}^{*}}{H}, 0\right) .
$$

And let $\Sigma(H)=\Sigma^{+}(H) \cup \Sigma^{-}(H)$. Then $\Sigma(H)$ has constant mean curvature $H$ globally. It is obvious that $\Sigma(H)$ has sphere topology and it is embedded.

From Lemma 3.4, we know $C^{-1} H^{-1} \leq l_{0} \leq C H^{-1}$. So it follows that for any compact set $K$, as long as $H$ is sufficiently small, $\Sigma(H)$ separates $K$ from infinity.

Now we calculate $|\Sigma(H)|$. Let $|\Sigma(H)|_{e}$ denote the area in Euclidean metric. We know that

$$
\left|\frac{|\Sigma(H)|_{e}}{|\Sigma(H)|}-1\right| \leq C l_{0}^{-1} \leq C H .
$$

So we need only to prove that $\left.\left|H^{2}\right| \Sigma(H)\right|_{e}-48 \pi \mid \leq C(p) H$. If we denote $g_{a^{\prime}(H)}$ as $g$ for short, we have

$$
H^{2}|\Sigma(H)|_{e}=16 \pi \int_{0}^{y_{5}^{*}} g \sqrt{1+g^{\prime 2}} d y .
$$

First we consider the integral on $\left[y_{2}^{*}, y_{4}^{*}\right]$. From (30) we know,

$$
H^{2} \leq \tau\left(g\left(y_{3}^{*}\right), g^{\prime}\left(y_{3}^{*}\right)\right) \leq C p H^{2}
$$

Then for some $C(p)>0, C H \leq g\left(y_{3}^{*}\right) \leq C(p) H, C(p) H^{-1} \leq g^{\prime}\left(y_{3}^{*}\right) \leq C H^{-1}$. 
There is $y_{6}^{*} \in\left[y_{3}^{*}, y_{4}^{*}\right]$ such that $g^{\prime}\left(y_{6}^{*}\right)=1$. We have

$$
\begin{array}{r}
\left|\int_{y_{6}^{*}}^{y_{4}^{*}} g \sqrt{1+g^{\prime 2}} d y-\int_{\Phi_{1}\left(y_{6}^{*}\right)}^{y_{4}^{*}} h(s) \sqrt{1+h^{\prime 2}(s)} d s\right| \leq C(p) H, \\
\left|\int_{\Phi_{2}\left(y_{6}^{*}\right)}^{\Phi_{1}\left(y_{6}^{*}\right)} h(s) \sqrt{1+h^{\prime 2}(s)} d s\right| \leq C(p) H, \\
\left|\int_{g\left(y_{3}^{*}\right)}^{g\left(y_{6}^{*}\right)} g \frac{\sqrt{1+g^{\prime 2}}}{g^{\prime}} d g-\int_{g\left(y_{3}^{*}\right)}^{g\left(y_{6}^{*}\right)} h \frac{\sqrt{1+h^{\prime 2}}}{h^{\prime}} d h\right| \leq C(p) H, \\
\left|\int_{y_{2}^{*}}^{y_{3}^{*}} g \sqrt{1+g^{\prime 2}} d y\right|,\left|\int_{y_{4}^{*}-1}^{\Phi_{2}\left(y_{3}^{*}\right)} h \sqrt{1+h^{\prime 2}} d y\right| \leq C(p) H,
\end{array}
$$

where the third one holds because

$$
\left|g\left(\frac{\sqrt{1+g^{\prime 2}}}{g^{\prime}}-\frac{\sqrt{1+h^{\prime 2}}}{h^{\prime}}\right)\right| \leq \frac{C(p) H^{2}}{g}+C(p) g H
$$

and the fourth one holds because $y_{3}^{*}-y_{2}^{*} \leq C(p) H$.

By direct calculation we know

$$
\int_{y_{4}^{*}-1}^{y_{4}^{*}} h \sqrt{1+h^{\prime 2}} d y=1 .
$$

So gathering all the inequalities we have

$$
\left|\int_{y_{2}^{*}}^{y_{4}^{*}} g \sqrt{1+g^{\prime 2}} d y-1\right| \leq C(p) H .
$$

In a similar way we can prove that

$$
\begin{gathered}
\left|\int_{0}^{y_{2}^{*}} g \sqrt{1+g^{\prime 2}} d y-1\right| \leq C(p) H, \\
\left|\int_{y_{4}^{*}}^{y_{5}^{*}} g \sqrt{1+g^{\prime 2}}-1\right| \leq C H .
\end{gathered}
$$

So we know

$$
\left|\int_{0}^{y_{5}^{*}} g \sqrt{1+g^{\prime 2}}-3\right| \leq C(p) H .
$$

So

$$
\left.\left|H^{2}\right| \Sigma(H)\right|_{e}-48 \pi \mid \leq C(p) H .
$$

However, if we revise Qing and Tian's proof of the uniqueness CMC spheres in 12. we know for a stable CMC sphere that separates the compact part from infinity and with $l_{0}$ large, $H^{2}|\Sigma|$ should be close to $16 \pi$. So the CMC spheres we constructed are unstable. 
Now we prove Theorem 1.2 Let's revise Lemma 4.7. Similarly we can choose $p<0$ small and $\delta(p)>0$ such that when $0<H<\delta(p)$ and $-\delta_{1}(H)<a-a^{\prime}(H)<$ 0 , we have $\left|g_{a}^{\prime}\left(y_{5}\right)\right|<\left|g_{a}^{\prime}\left(y_{1}\right)\right|$. So we can choose a proper $p=p(H)$ such that $\left|g_{a}^{\prime}\left(y_{5}\right)\right|=\left|g_{a}^{\prime}\left(y_{1}\right)\right|$. In this case, we have

$$
\lim _{a \rightarrow a^{\prime}(H)^{-}} g_{a}^{\prime}\left(y_{5}\right)=\lim _{a \rightarrow a^{\prime}(H)^{-}} g_{a}^{\prime}\left(y_{1}\right)=-\infty .
$$

Now we can analyze the behavior of $g_{a}$ on $y_{1}$ and $y_{3}$ by using the same method as used in Lemma 4.8. We will get a singular limit of three spheres (each one is embedded). They share the same axis and the central one meets its two neighbors at two poles where $x_{2}=x_{3}=0$. By using the same method as used in Lemma 4.9. we know all the three spheres are smooth and have constant mean curvature $H$.

The problem is, for different $H$, we may get different $p(H)$ (which is bounded independent of $H$ ). Note that for a particular $H>0$, when we do all the constructions above, only the metric in a domain

$$
D(H)=\left\{\left(x_{1}, x_{2}, x_{3}\right) \mid C^{-1} H^{-1} \leq \sqrt{x_{1}^{2}+x_{2}^{2}+x_{3}^{2}} \leq C H^{-1}\right\}
$$

really matters. If we have constructed $\Sigma_{n}^{i}, i=1,2,3$ for some $H_{n}>0$, we can choose a much larger scale to construct $\Sigma_{n+1}^{i}, i=1,2,3$. We may assume that $D\left(H_{i}\right), D\left(H_{j}\right)$ are disjoint for $i \neq j$. We choose $p$ as a smooth function of $l=$ $\sqrt{x_{1}^{2}+x_{2}^{2}+x_{3}^{2}}$ such that $p=p_{i}$ in each $D\left(H_{i}\right)$. We can assume that $D\left(H_{i}\right)$ is far away from $D\left(H_{i+1}\right)$ and $p^{\prime}(l)$ is small enough such that the $\phi_{\lambda, p}$ term does not influence the mass. In this way, we get a smooth asymptotically Schwarzschild metric with mass 1 . Then we can prove Theorem 1.2 .

\section{References}

[1] S. Brendle. Constant mean curvature surfaces in warped product manifolds. Publ. Math-Paris., 117(1):247-269, 2013.

[2] S. Brendle and M. Eichmair. Large outlying stable constant mean curvature spheres in initial data sets. Invent. Math., 197(3):663-682, 2014.

[3] A. Carlotto, O. Chodosh, and M. Eichmair. Effective versions of the positive mass theorem. arXiv:1503.05910v1, 2015.

[4] L.H. Huang. Foliations by stable spheres with constant mean curvature for isolated systems with general asymptotics. Comm. Math. Phys., 300(2):331$373,2010$.

[5] G. Huisken and S.T. Yau. Definition of center of mass for isolated physical systems and unique foliations by stable spheres with constant mean curvature. Invent. Math., 124:281-311, 1996. 
[6] S. Ma. Uniqueness of the foliation of constant mean curvature spheres in asymptotically flat 3-manifolds. Pac. J. Math., 252(1):145-179, 92011.

[7] S. Ma. On the radius pinching estimate and uniqueness of the $\mathrm{cmc}$ foliation in asymptotically flat 3-manifolds. Adv. Math., 288:942-984, 2016.

[8] S. Ma and F. Pacard. Constant mean curvature surfaces of delaunay type along a closed geodesic. arXiv:1412.4240, 2014.

[9] C. Nerz. Foliations by stable spheres with constant mean curvature for isolated systems without asymptotic symmetry. arxiv:1408.0752v2.

[10] A. Neves and G. Tian. Existence and uniqueness of constant mean curvature foliation of asymptotically hyperbolic 3-manifolds. Geom. Funct. Anal., 19(3):910-942, 122009.

[11] A. Neves and G. Tian. Existence and uniqueness of constant mean curvature foliation of asymptotically hyperbolic 3-manifolds ii. J. Reine. Angew. Math., 641:69-93, 2010.

[12] J. Qing and G. Tian. On the uniqueness of the foliation of spheres of constant mean curvature in asymptotically flat 3-manifolds. J. Am. Math. Soc., (4):1091-1110, 2007. 